\title{
The chromatin modification by SUMO-2/3 but not SUMO-1 prevents the epigenetic activation of key immune-related genes during Kaposi's sarcoma associated herpesvirus reactivation
}

Pei-Ching Chang ${ }^{1 *}$, Chia-Yang Cheng ${ }^{2,3}$, Mel Campbell ${ }^{4}$, Yi-Cheng Yang ${ }^{1}$, Hung-Wei Hsu' ${ }^{1}$, Ting-Yu Chang ${ }^{1}$, Chia-Han Chu' ${ }^{2}$, Yi-Wei Lee ${ }^{1}$, Chiu-Lien Hung ${ }^{5}$, Shi-Mei Lai ${ }^{2}$, Clifford G Tepper ${ }^{4,6}$, Wen-Ping Hsieh', Hsei-Wei Wang ${ }^{1}$, Chuan-Yi Tang ${ }^{3}$, Wen-Ching Wang ${ }^{2}$ and Hsing-Jien Kung ${ }^{4,5,6,88^{*}}$

\begin{abstract}
Background: SUMOylation, as part of the epigenetic regulation of transcription, has been intensively studied in lower eukaryotes that contain only a single SUMO protein; however, the functions of SUMOylation during mammalian epigenetic transcriptional regulation are largely uncharacterized. Mammals express three major SUMO paralogues: SUMO-1, SUMO-2, and SUMO-3 (normally referred to as SUMO-1 and SUMO-2/3). Herpesviruses, including Kaposi's sarcoma associated herpesvirus (KSHV), seem to have evolved mechanisms that directly or indirectly modulate the SUMO machinery in order to evade host immune surveillance, thus advancing their survival. Interestingly, KSHV encodes a SUMO E3 ligase, K-bZIP, with specificity toward SUMO-2/3 and is an excellent model for investigating the global functional differences between SUMO paralogues.

Results: We investigated the effect of experimental herpesvirus reactivation in a KSHV infected B lymphoma cell line on genomic SUMO-1 and SUMO-2/3 binding profiles together with the potential role of chromatin SUMOylation in transcription regulation. This was carried out via high-throughput sequencing analysis. Interestingly, chromatin immunoprecipitation sequencing (ChIP-seq) experiments showed that KSHV reactivation is accompanied by a significant increase in SUMO-2/3 modification around promoter regions, but SUMO-1 enrichment was absent. Expression profiling revealed that the SUMO-2/3 targeted genes are primarily highly transcribed genes that show no expression changes during viral reactivation. Gene ontology analysis further showed that these genes are involved in cellular immune responses and cytokine signaling. High-throughput annotation of SUMO occupancy of transcription factor binding sites (TFBS) pinpointed the presence of three master regulators of immune responses, IRF-1, IRF-2, and IRF-7, as potential SUMO-2/3 targeted transcriptional factors after KSHV reactivation.
\end{abstract}

Conclusion: Our study is the first to identify differential genome-wide SUMO modifications between SUMO paralogues during herpesvirus reactivation. Our findings indicate that SUMO-2/3 modification near protein-coding gene promoters occurs in order to maintain host immune-related gene unaltered during viral reactivation.

\footnotetext{
* Correspondence: pcchang@ym.edu.tw; hkung@nhri.org.tw

${ }^{1}$ Institute of Microbiology and Immunology, National Yang-Ming University, Taipei 11221, Taiwan

${ }^{4}$ UC Davis Cancer Center, University of California, Davis, CA 95616, USA

Full list of author information is available at the end of the article
} 


\section{Background}

SUMOylation was initially identified as a reversible posttranslational modification that controls a variety of cellular processes, including cellular signal transduction, replication, chromosome segregation, and DNA repair [1-3]. The growing list of Small Ubiquitin-like MOdifier (SUMO) substrates includes transcription factors and epigenetic regulators, which implies the involvement of the SUMO modification system in the epigenetic regulation of gene expression [4] and in the initiation and maintaining of heterochromatin silencing [5,6]. SUMO has been found in all eukaryotes but is not present in prokaryotes. The global regulatory role of SUMOylation in gene expression and protein interaction has been richly explored in lower eukaryotes such as yeast $[7,8]$. However, there is only a single SUMO protein in yeast, whereas there are three major protein conjugating isoforms present in mammals; these are SUMO-1, and the highly similar SUMO-2 and SUMO-3, which are often refer to as SUMO-2/3. Recent reports have pinpointed some important differences between SUMO-1 and SUMO-2/3. These are, firstly, that SUMO-1 is conjugated to its substrates as a mono-SUMOylation, whereas SUMO-2/3 are able to form poly-SUMOylation chains [9]. Moreover, SUMO-1 acts like a chain terminator to the SUMO-2/3 polymers [10]. Secondly, inside cells, SUMO-1 appears mostly conjugated to proteins, whereas SUMO-2/3 are primarily found in the free form and are increased in conjugation to substrates when there are cellular stresses $[11,12]$. Thirdly, the kinetics of SUMO-1 de-conjugation is slower than that of SUMO$2 / 3$ [13]. Fourthly, a preferential association of SUMO-1 with the nuclear envelope and nucleolus, whereas SUMO-2/3 are distributed throughout the nucleoplasm [12]. Fifthly, although many substrates can be modified by both SUMO-1 and SUMO-2/3, some substrates are preferentially modified by one SUMO isoform or the other. The underlying complexity of SUMOylation has been extended by the identification of non-covalent interaction with effectors via SUMO interaction motifs (SIMs) [14]. SIMs are critical to both SUMO conjugation and SUMO-mediated effects. Structure analysis shows the potential differential specificity of SIMs toward SUMO paralogues [15]. The specificity of the SIM in relation to the SUMO E3 ligase [16-18] and substrate [19] has been found to control SUMO paraloguespecific modification. Consequentially, this provides an additional interaction platform for the selective recruitment of SUMO-1 or SUMO-2/3 specific SIM-containing effector proteins. While numerous studies have provided considerable insight into the differences in specificity between SUMO paralogues, their scope has been usually limited to a single host factor in each case. Discerning the genome-wide chromatin modification by SUMO paralogue during herpesvirus reactivation will greatly advance our knowledge of their differential role in epigenetic regulation and pathogenesis.

Due to the functional flexibility and far-reaching downstream consequences of SUMO, viruses have evolved different strategies that are able to manipulate the SUMO pathway and improve their survival [20-25]. This makes SUMO a potential target for antiviral therapy. Most current knowledge related to SUMO modification and viruses has been obtained from studying DNA tumor viruses, especially members of the herpesviridae and have been inevitably linked to counteracting the host's antiviral properties. SUMOylation has been found to affect most of the immediate-early and early proteins of herpesviruses, which are usually transcriptional factors. BZLF1 and Rta of Epstein-Barr virus (EBV) [26-29], and the K-bZIP of KSHV are three such examples [25]. Viruses are also able to directly target the key enzymes of the SUMOylation pathway, namely the SUMO E1 activating enzyme, Aos1/ Uba2, the SUMO E2 enzyme, Ubc9, the SUMO E3 ligases, and the SUMO protease SENP/Ulp; this allows the virus to take charge of the SUMOylation modulating factors in the cell [30]. Recently, we identified the first viral SUMO E3 ligase, KSHV K-bZIP; this enzyme has specificity toward SUMO-2/3 [16]. The encoding of a SUMO-2/3 specific viral SUMO E3 ligase by KSHV suggests that, potentially, KSHV is able to exploit the SUMO pathway to globally regulate viral and host transcriptional programs. This, in turn, implies that SUMO-2/3 may function in a manner that is distinct from SUMO-1 during viral reactivation.

$\mathrm{KSHV}$, also known as human herpesvirus type 8 , is a $\gamma$-herpesvirus associated with Kaposi's sarcoma (KS), primary effusion lymphomas (PEL) and multicentric Castlemen's disease [31]. It is one of the seven recognized human cancer viruses [32]. Like all herpesvirus, KSHV has distinct latent and lytic phases. Establishment of latency is a common property of herpesvirus in infected cells and is able to prevent their elimination by the host immune response, to maintain life-long infection, and to induce tumorigenesis $[33,34]$. In order to establish infection and maintain latency, KSHV has acquired a series of different strategies that are able to limit innate antiviral responses and evade host immune surveillance, thus allowing the persistence of infection. For example, KSHV dedicates a large portion of its genome to encoding cellular homologues of host immune modulators and is able to express unique viral proteins that have immunomodulatory roles $[35,36]$. For instance, KSHV-replication and transcriptional activator (K-Rta), an immediate early (IE) protein of KSHV, which is able to activate a wide spectrum of KSHV lytic genes and thereby alone can induce viral reactivation, has been found to block the interferon (IFN) pathway by targeting interferon regulatory factor (IRF) for degradation. The KSHV-encoded basic leucine zipper 
protein (K-bZIP), one of the earliest viral protein expressed right after K-Rta during acute infection and viral reactivation, has also been found to inhibit the IFN pathway by direct impeding IRF binding to the IFN promoter $[37,38]$. The IFN pathway has also been found to be repressed by K-bZIP in a SUMOylation-dependent manner [39]. Moreover, recent studies have shown that SUMOylation of the IRFs occurs during viral infection and these changes are essential to allowing the virus to negatively regulate the IFN pathway [24,40,41]. Another strategy employed by herpesviruses such as HSV-1, the prototypical member of the Herpesviridae, is the complete suppression of cellular gene expression, a process termed host shutoff. This phenotype is found during lytic herpesviral infection and is believed to play an important role in establish herpesviral latency [42]. In HSV-1, the global shutoff of host gene expression occurs via two major and distinct inhibitory pathways. One is a global increase in the rate of mRNA degradation and the other is a virusinduced suppression of host mRNA synthesis [43]. For $\mathrm{KSHV}$, mRNA degradation is performed by the host shutoff factor SOX [44]. The linking of SUMOylation to transcription repression and the finding that K-bZIP is a SUMO-2/3 specific E3 ligase led us to examine the possibility that there may be global silencing of host genes by K-bZIP. We have reported previously that K-bZIP, when overexpressed, was indeed a general gene-silencer [45].

To gain a better understanding of the differential functionality of SUMO-1 and SUMO-2/3 conjugation on chromatin in transcriptional regulation of host genes during KSHV reactivation, we performed a genome-wide mapping of chromatin modification by SUMO paralogues using ChIP-seq, a technology that allows the direct identification of all SUMO binding sites on the genome. Here, we demonstrate that the chromatin-binding patterns for SUMO-1 and SUMO-2/3 are very similar in the non-reactivated control cells. Interestingly, during viral reactivation, distinct dynamic chromatin-binding of SUMO paralogues was observed. We have demonstrated that the chromatin occupancy of SUMO-2/3 but not of SUMO-1 is significantly increased during viral reactivation and this enrichment is not randomly distributed. Enrichment occurs in promoter regions where transcription factors binds. Potential SUMO-2/3 target TFs on the chromatin were identified by annotating SUMO peaks in relation to putative transcription factor binding sites (TFBS) using the Transfac Matrix Database. Here, we provide the first comprehensive profile that compares the SUMO-1 and SUMO-2/3 landscapes in the human genome and predicts the relevant potential modifying TFs that bind to the chromatin. Previous findings from yeast study have shown that SUMO is globally associated with transcriptionally active genes $[7,46]$ and facilitates the shutting off of induced gene transcription [7].
This suggests that SUMO modification may also play a global role in transcription regulation in mammals. Large scale comparative analysis of ChIP-seq and transcriptome studies using RNA-seq in this study indicates that both SUMO-1 and SUMO-2/3 label the promoters of highly active genes in the non-reactivated control cells. However, during KSHV reactivation, the SUMO-2/3 modifications are greatly enriched in the promoters of highly active genes that show little change in gene expression.Together with previous findings from other studies, our results indicate that SUMO- 1 and SUMO-2/3 may play similar roles in maintaining the expression of highly transcriptional active genes in non-reactivated cells. However, the enrichment of SUMO-2/3 at transcriptionally active genes that show no change in expressional level during viral reactivation suggests that SUMO-2/3, but not SUMO-1, ensures the steady-state expression of host genes without overt activation during viral reactivation. Consistent with studies exploring the functional analysis of SUMO paralogues in specific protein molecules such as Daxx [47], in the present study we demonstrate that there are distinct differences in the global roles of SUMO-1 and SUMO-2/3 in cells that are under stress, such as when there is herpesvirus reactivation.

\section{Methods \\ Cell culture}

The TREx-Flag(x3)-His(x3)-K-Rta BCBL-1 cell line (TREx-F3H3-K-Rta BCBL-1) and TREx-Flag(x3)-His (x3)-K-bZIP BCBL-1 cell line (TREx-F3H3-K-bZIP BCBL-1) were grown in RPMI 1640 containing 15\% FBS, $50 \mu \mathrm{g} / \mathrm{ml}$ blasticidin and $100 \mu \mathrm{g} / \mathrm{ml}$ hygromycin (Invitrogen, Carlsbad, CA). To induce $\mathrm{KSHV}$ reactivation, $0.2 \mu \mathrm{g} / \mathrm{ml}$ doxycycline (dox) was added to the TREx-F3H3-K-Rta BCBL-1 cells. K-Rta expression was confirmed by Western blot analysis.

The shRNA cassette of SUMO-2 (5'-CACCGAGGCA GATCAGATTCCGAT TCGAAAATCGGAATCTGATC TGCCTC-3') and SUMO-3 (5'-CACCGGATGAA TC TGTAACTTAACGAATTAAGTTACAGATTCATCC-3') was inserted into pLenti4-H1/TO shRNA plasmid and introduced into TREx-F3H3-K-Rta BCBL-1 cells by lentiviral transduction. Cells were selected for 14 days by 300 $\mu \mathrm{g} / \mathrm{ml}$ zeocine (InvivoGen, ant-zn-1) and purified by Ficoll. Knockdown efficiency of SUMO-2/3 by shRNA were tested by treated the cells with doxycycline (Dox) for 24 and 48 hours. TREx-F3H3-K-Rta-shSUMO-2/3 BCBL-1 cells were maintained as described for TREx-F3H3-K-Rta BCBL-1 and supplemented with $300 \mu \mathrm{g} / \mathrm{ml}$ zeocine.

\section{Plasmid construction}

The full-length human IRF-1, IRF-2 and IRF-7 cDNAs were obtained by reverse-transcription polymerase chain 
reaction (RT-PCR) using an RNA sample isolated from BCBL-1 and using an Oligo(dT) primer. The primers $5^{\prime}$ AAACGGTCCGATGCCCATCACTCGGATG-3' and 5' AAACGGACCGCTACG GTGCACAGGGAAT-3' were used for RT-PCR and cloning of IRF-1, the primers 5'AAACGGTCCGATGCCGGTGGAAAGGATG-3' and 5' AAACGGACCGTTAA CAGCTCTTGACGCG-3' were used for RT-PCR and cloning IRF-2, and the primers $5^{\prime}$ AAACGGTCCGATGGCCTTGGCTCCTGAG-3' and 5' AAACGGACCGCTAG GCGGGCTGCTCCAG-3' were used for RT-PCR and cloning IRF-7. The IRF DNA fragment were then cloned into pcDNA3 and confirmed by DNA sequencing.

\section{Chromatin immunoprecipitation-sequencing (ChIP-Seq), ChIP-reChIP assay and real-time quantitative PCR (qPCR)} After 12 hours of K-Rta induction to allow KSHV reactivation in the TREx-F3H3-K-Rta BCBL-1 cell line, $1 \times 10^{7}$ cells were harvested. ChIP assays were performed following the protocol described by the Farnham laboratory (provided at http://genomics.ucdavis.edu/farnham). ChIPreChIP assays were performed by Re-ChIP-IT kit (Active Motif, Carlsbad, CA) following the manufacturer's instruction. ChIP grade rabbit polyclone antibodies specific against SUMO-1 [Y299] (Abcam, ab32058) and against SUMO-2/3 (Abcam, ab3742), as well as rabbit non-immune serum IgG (Alpha Diagnostic International, San Antonio, TX), were used for the ChIP and ChIP-reChIP assays. Rabbit monoclone antibodies specific against IRF-7 (GeneTex, Irvine, CA) was used for the ChIP-reChIP assays.

For ChIP-Seq assay, ChIPed DNA was prepared from $5 \times 10^{7}$ cells that had been resuspended in $30 \mathrm{ul}$ of $\mathrm{ddH}_{2} \mathrm{O}$ and ChIP-seq library construction was then carried by following the sample preparation protocol from Illumina. Short reads (100 bp) from both ends (paired-end sequencing) of size-selected (400 bp) DNA fragments were selected and subjected to high throughput sequencing on an Illumina Genome Analyzer ${ }_{I I}$ System. The ChIP-Seq data was aligned onto the human genome hg19 build using UCSC. Around $6 \times 10^{7}$ reads were mapped for each sample after filtering and quality control (QC) were carried out. In this study we used the enriched region detection method of Avadis NGS (Strand Scientific Intelligence, San Francisco, CA) to localize potential protein binding sites in order to delineated the SUMO- 1 and SUMO-2/3 binding patterns.

The binding sites were verified by $\mathrm{SYBR}^{\circ}$ Green Based qPCR using a CFX connect ${ }^{\text {tix }}$ real-time PCR detection system (Bio-Rad, Richmond, CA). Specific primer sets were designed around the identified binding sites for this purpose.

\section{RNA-seq and RT-qPCR analysis}

Total RNA was harvested using TRIzol reagent (Invitrogen, Carlsbad, CA) from TREx-F3H3-K-Rta BCBL-1 at 12 and
24 hours after K-Rta induced viral reactivation according to the manufacturer's instructions. RNA-seq was conducted at the Sequencing Core of National Research Program for Genomic Medicine at National Yang-Ming University VYM Genome Research Center using an Illumina Genome Analyzer $_{I I}$. Sequencing reads were first trimmed with human ribosomal RNA sequences (28S, 18S, 5S, human ribosomal DNA complete repeating unit and mitochondrial ribosomal RNA) by Bowtie (version 1.0.0) with default parameters and then aligned the high quality reads to human reference genome hg19 using TopHat (version 2.0.8b) with Bowtie version 2.1.0 and samtools (version 0.1.9) with transcriptome information obtained from Ensembl Release 70 and NonCode v3.0. The transcript abundances were estimated in fragments per kilobase of transcript per million mapped reads (FPKM) by Cufflinks version 2.1.1. Genes from all three samples with FPKM $>0.05$ were considered to be expressed and were used for the remaining analysis. Differential gene expression of the samples (K-Rta induction for 12 and 24 hours $v s$. control) was analyzed by comparing FPKM. For RT-PCR, $2 \mu \mathrm{g}$ of total RNA was reversetranscribed using SuperScript ${ }^{\text {tm }}$ III First-strand synthesis system (Invitrogen) and Oligo-dT. qPCR was carried out based on the manufacturer's protocol (iQ SYBR Green Supermix, Bio-Rad).

\section{Immunoprecipitation and western blot analysis}

Transfected $293 \mathrm{~T}$ cells were collected in modified radioimmune precipitation assay (RIPA) buffer (50 mM Tris$\mathrm{HCl}$ [pH 6.7], 1\% NP-40, 0.25\% sodium deoxycholate, 150 $\mathrm{mM} \mathrm{NaCl}, 1 \mathrm{mM}$ EDTA) supplemented with $1 \mathrm{X}$ protease inhibitor cocktail (Roche, Penzberg, Germany). TCLs were incubated with anti-FLAG M2-agarose (Sigma-Aldrich, St. Louis, $\mathrm{MO}$ ) overnight at $4^{\circ} \mathrm{C}$. Immune complexes were captured by protein A and protein G Sepharose beads. Beads were washed, and the bound proteins were analyzed by immunoblotting. Antibodies used for immunoblotting were anti-IRF-1 (Cell Signaling Technologies, Beverly, MA), anti-IRF-2 (Cell Signaling Technologies), anti-IRF-7 (GeneTex), anti-SUMO-2/3 antibodies.

\section{Results}

Global identification of the chromatin binding patterns of SUMO paralogues reveals that KSHV reactivation is associated with specific enrichment of SUMO-2/3

SUMO modifications of transcription regulatory proteins and chromatin modifying enzymes are linked to the epigenetic regulation of gene transcription. SUMO-1 and SUMO-2/3 have both common and distinct substrates, but their global functional roles in epigenetic regulation have not as yet been fully investigated. As mentioned earlier, DNA viruses have evolved different strategies that allow them to manipulate the SUMO pathway in a 
manner that helps their survival. We previously identified a KSHV lytic protein, K-bZIP, as a viral SUMO E3 ligase with specificity toward SUMO-2/3 [16]. Using KSHV as a model in conjunction with ChIP-seq to interrogate the binding sites of the various SUMO paralogues during viral reactivation, we hoped to distinguish the epigenetic regulatory role of the SUMO isoforms during viral replication. KSHV is a particularly attractive model as its reactivation can be switched on by the expression of a single K-Rta gene, and a well characterized Doxinducible TREx-F3H3-K-Rta BCBL-1 cell line is available for this purpose. This study has the ability to pinpoint the global functional differences in terms of epigenetic regulation of the SUMO isoforms during viral pathogenesis.

To study the epigenetic regulation of SUMO paralogues in association with KSHV reactivation, the genome-wide in vivo binding sites of SUMO-1 and SUMO-2/3 were analyzed using massively parallel chromatin immunoprecipitation in combination with high throughput sequencing (ChIP-Seq); these processes were carried out on a K-Rtainducible KSHV infected primary effusion lymphoma (PEL) cell line, TREx-F3H3-K-Rta BCBL-1. Chromatin samples from TREx-F3H3-K-Rta BCBL-1 cells before and after K-Rta induction to allow KSHV reactivation were isolated and subjected to the ChIP assay using ChIP grade SUMO-1 and SUMO-2/3 antibodies. High-throughput sequencing was then performed to measure the binding of SUMO-1 and SUMO-2/3 from a single run of ChIP assay. Approximately the same number $\left(6 \times 10^{7}\right)$ of reads from the KSHV un-induced and induced samples were mapped to the human reference genome, hg19. Using an enrichment peak calling algorithm, we found a total of 31315 and 45846 high confidence SUMO-1 and SUMO-2/3 enrichment regions, respectively, in the non-reactivated control cells. After K-Rta induction for 12 hours, a total of 39626 SUMO-1 enrichment regions (an increase in $8 \mathrm{~K}$ peaks of $\sim 1.3$-fold compared to the control cells) and 86479 high confidence SUMO-2/3 enrichment regions (an increase in $40 \mathrm{k}$ peaks of $\sim 1.9$-fold compared to the control cells) were identified (Figure 1A). Consistent with our previous findings showing that KSHV encodes a SUMO-2/3 specific E3 ligase in its lytic phase [16], there was a significant increase in SUMO-2/3 modification across human genome, whereas SUMO-1 modification showed a relatively similar occupancy abet with a slight increase; these findings, suggest that KSHV specifically exploits SUMO-2/3 in order to regulate viral and host transcriptional programs.

Common binding sites that were shared by the SUMO paralogues were assessed by examining the overlap in their binding profiles between the K-Rta induced and noninduced states. The results showed that around $30 \%$ of the SUMO-1 and SUMO-2/3 binding sites under both conditions showed colocalization (Figure 1B). Interestingly, the number of SUMO-2/3 specific binding sites was increased by $12 \%$ (from $\sim 27 \mathrm{k}$ to $\sim 57 \mathrm{k}$ ) while the number of SUMO1 specific binding sites was decreased by $11 \%$ (from $\sim 13 \mathrm{k}$ to $\sim 10 \mathrm{k}$ ) during viral reactivation (Figure 1B). These findings suggest that KSHV reactivation is accompanied by significant changes in the magnitude of SUMO-2/3 tagging across the genome. By contrast, the level of SUMO-1 tagging on chromatin remains relatively unchanged.

\section{SUMO-2/3 is enriched on the promoter regions after KSHV reactivation}

SUMO is capable of binding to chromatin by modifying chromatin remodeling proteins and transcription

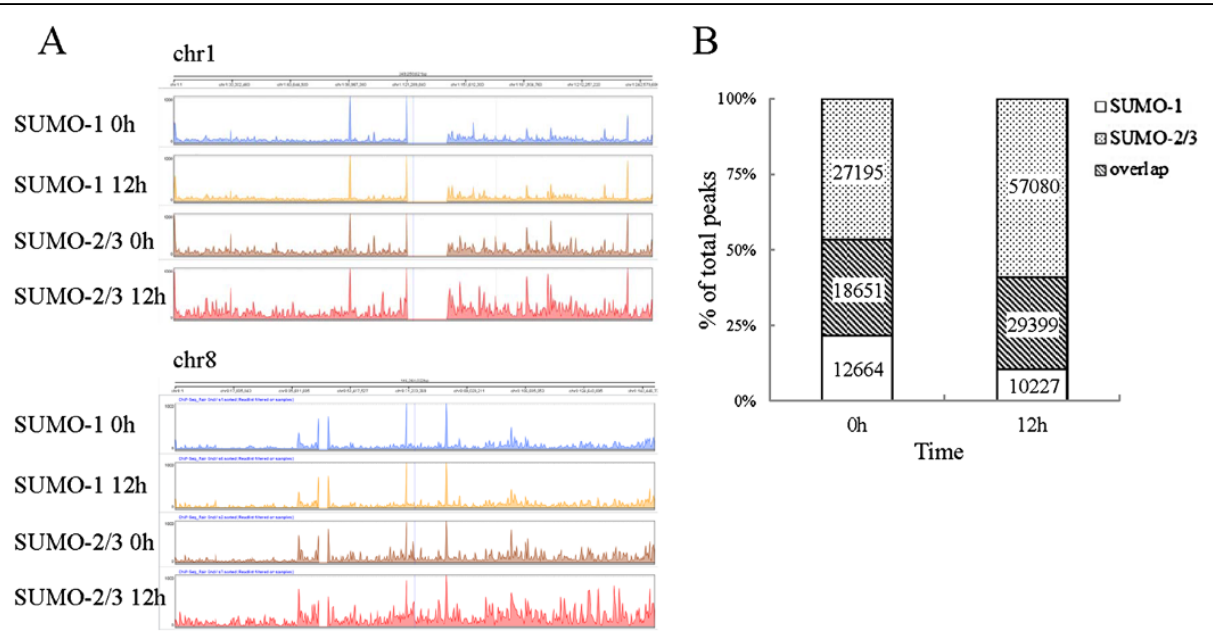

Figure 1 Overview of ChIP-seq data showing chromatin occupancy of SUMO paralogues during KSHV reactivation. (A) Histograms of ChIP-seq profiles across chromosome 1 and 8 for SUMO- 1 and SUMO-2/3 binding sites before and after KSHV reactivation. (B) Overlap of SUMO-1 and SUMO-2/3 binding sites before and after KSHV reactivation in BCBL-1 cells; numbers indicate counts for overlapping and non-overlapping peaks. 
factors [48]. In this context, SUMO modifications are likely to have specific distributions across the genome. As expected, SUMO target sites occur across all chromosomes but are not randomly distributed. They are enriched in regions containing genes, notably in regions annotated as promoters. As Figure 2A reveals, chromatin-bound SUMO paralogues are commonly centered and symmetrically distributed within 500 bp around transcription start sites (TSSs). This pattern is similar to that reported for chromatin modification by SUMO-1 through the cell cycle [46]. Interestingly, after overlaying the SUMO-binding data after viral reactivation onto the control SUMObinding data, we discovered that there was a significant increase in SUMO-2/3 occupancy near to TSSs (Figure 2C) after KSHV reactivation, whereas SUMO-1 occupancy showed a slight decrease (Figure 2B). A similar pattern was identified for the groups of peaks that contain only SUMO-1 or SUMO-2/3 specific modification.

Consistent results were obtained when SUMO peaks were normalized for the size of defined genome compartment. SUMO paralogues showed a relative higher peak density in promoter regions (TSSs $\pm 500 \mathrm{bp}$ ), whereas the binding to the gene bodies themselves (transcribed regions), the transcription end sites (TESs), the regions upstream of the gene, the regions downstream of the gene and the intergenic regions were low (Figure 2D and 2E). Consistently, the peak density of SUMO-2/3 (Figure 2E), but not of SUMO-1 (Figure 2D), was significantly increased in the promoter regions during viral reactivation. These findings indicate that, while the chromatin-bound SUMO paralogues are both centered on the TSSs, only chromatin-bound SUMO-2/3 is significantly increased during KSHV reactivation.

\section{Global prediction of potential SUMO-1 and SUMO-2/3} targeting of chromatin-bound transcription factors

Typical SUMO binding sites are focal and consist of no more than a few hundred base pairs, a pattern reminiscent of the "peaks" associated with transcription factors. A large number of known SUMO conjugates in mammals are transcription factors. To predict the potential chromatin-associated transcription factors (TFs) that are SUMOylated, we annotate SUMO target sites within promoter regions in relation to transcription factor

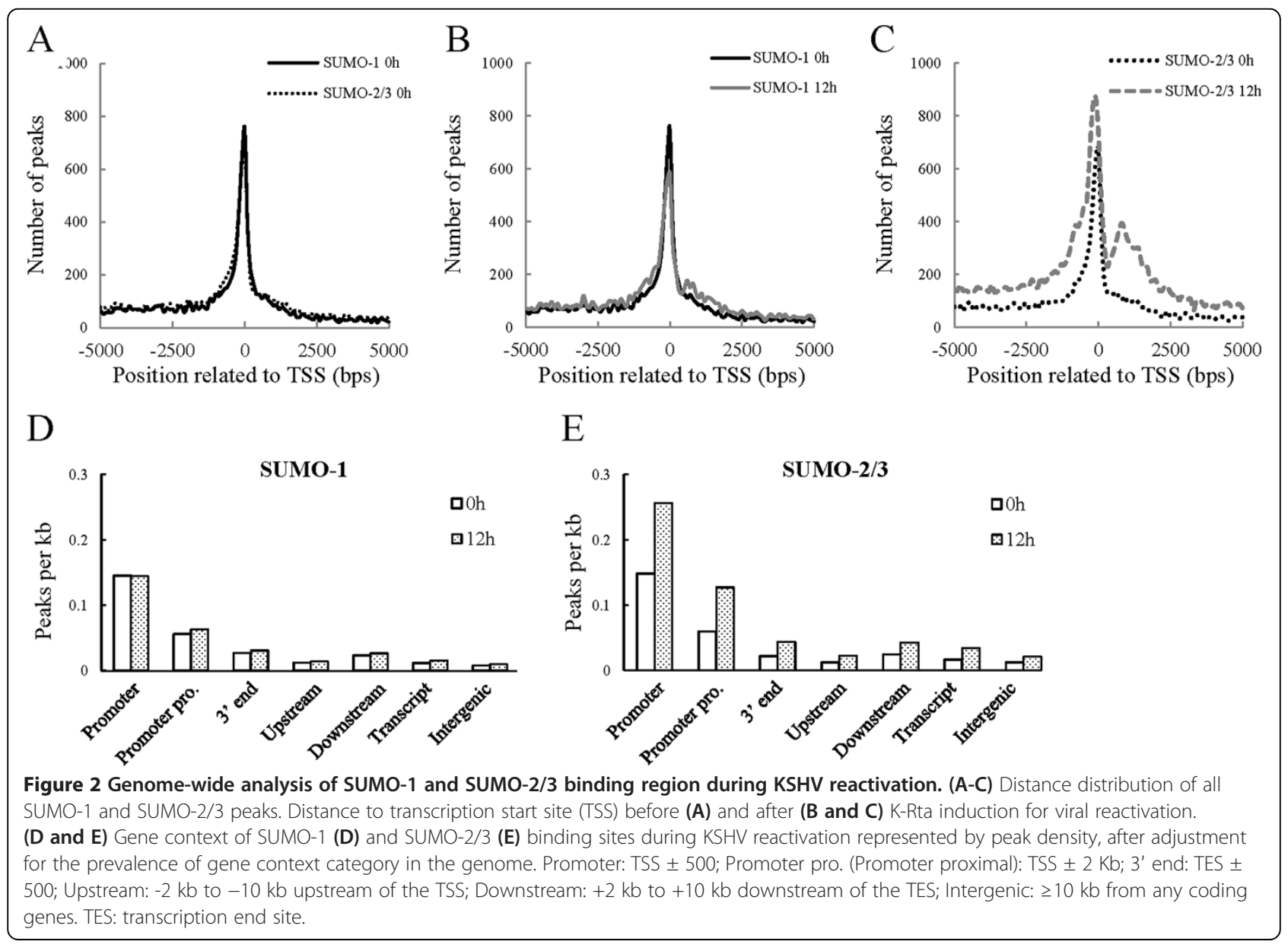


binding sites (TFBS) from the Transfac Matrix Database (v7.0) created by Biobase. This database contains 258 TFBS weight matrices that represent the potential DNA binding sites of 176 TFs across the genome. The SUMO enriched peaks for each TFBS in the promoter region were normalized with their own distribution frequency. Potential SUMO target TFs were ranked by percentage and Hampel Identifier was used to identify the TFBSs that were significantly correlated with SUMO binding [49]. The number of SUMO peaks identified before viral reactivation was used as the control. High confidence TFBSs that correlated with SUMO-1 and SUMO-2/3 peaks were mapped and are represented here as "potential SUMO-1 and SUMO-2/3 target TFs". Interestingly, during viral reactivation, the SUMO-1 target TFs decreased from 18 to 10 , while the SUMO-2/3 target TFs were significantly increased from 22 to 86 (Figure 3A). When we overlapped the potential SUMO-1 and SUMO- $2 / 3$ target TFs, we found that $74 \%$ of the TFs shared by SUMO paralogues in the non-reactivated control cells and this decreased (10\%) during viral reactivation (Figure 3A). Around $20 \sim 30 \%$ of the potential SUMO-1 and SUMO-2/3 target TFs overlapped before and after KSHV reactivation (Figure 3B). Moreover, SUMO-1 target TFs consisted of more non-overlapping TFs before viral reactivation (11 of 21 ), while, on the other hand, there were more non-overlapping TFs for SUMO-2/3 (65 of 87) that were recognized after viral reactivation (Figure 3B). Collectively, these results suggest that SUMO-2/3 significantly increased its tagging of TFs bound to promoter regions during KSHV reactivation.

The top twenty potential SUMO-1 and SUMO-2/3 target TFs before and after KSHV reactivation are listed in Tables 1, 2, 3 and 4. If less than twenty TFs have been identified, all of them are listed. One interesting point involves the reasons for SUMO-2/3 target TFs iden- tification after viral reactivation, which is quite different from that for SUMO-1 target TFs. As shown in Table 4, there is a more significant increase in peak numbers for the top-20 SUMO-2/3 target TFs after KSHV reactivation comparing with the top twenty TFs listed from the nonreactivated control cells (Table 2). In contrast, the peak number for the SUMO-1 target TFs after viral reactivation almost all decreased (Table 3); this decrease is less than that of the control (Table 1). The findings indicate an increase in SUMO-2/3 modification of TFs during viral reactivation. In contrast, SUMO-1 modification of TFs after viral reactivation had decreased. These finding suggest that SUMO paralogues are differentially regulated in a global manner under certain circumstances, for example when there is viral reactivation as is the case here. The SUMO- 1 and SUMO-2/3 specific TFBSs identified here provides a framework that allows the study of the potential functional differences between SUMO paralogues.

\section{Identification of potential transcription factors targeted by SUMO-2/3 during KSHV reactivation}

The presence of highly enriched SUMO-2/3 binding sites around the promoter regions during viral reactivation suggest that SUMO-2/3 might be directly or indirectly targeting a large group of transcription factors during KSHV reactivation. In order to pinpoint the most important gene-regulating TFs that are targeted by SUMO-2/3 during viral reactivation, we collected genes with SUMO-2/3 targeted TFBSs at their promoter before and after viral reactivation and group them into an up-group (SUMO peaks increase $>1.5 \mathrm{X}$ ), a down-group (SUMO peaks decrease $>1.5 \mathrm{X}$ ) and a no-change-group (SUMO peaks variants within 1.5X). When we ranked the TFs by total gene number, the top 10 most important gene-regulating TFs targeted by SUMO-2/3 after KSHV reactivation could be identified (Figures 4 and 5).

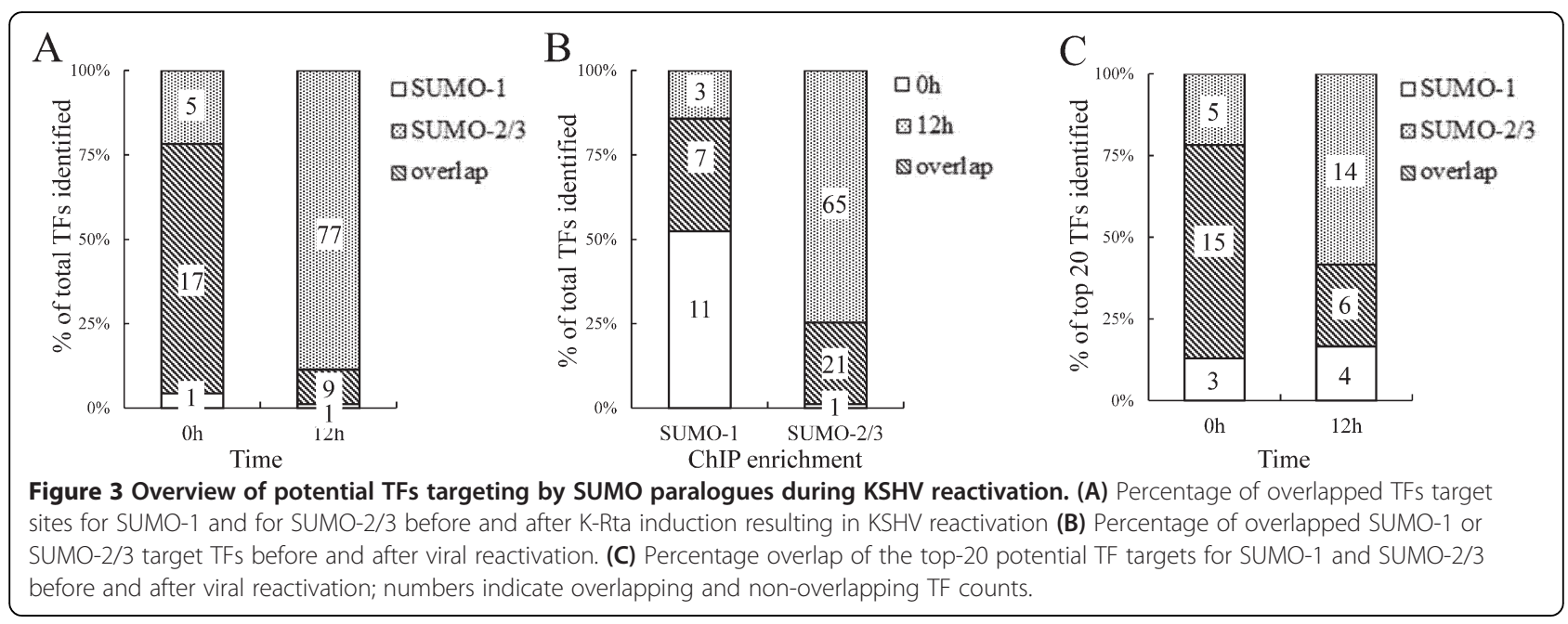


Table 1 Top 20 potential SUMO-1 targeting TFs before KSHV reactivation

\begin{tabular}{|c|c|c|c|c|c|c|c|c|c|}
\hline \multirow[t]{2}{*}{ Rank } & \multirow[t]{2}{*}{ Name } & \multirow[t]{2}{*}{ Transfac } & \multirow[t]{2}{*}{$\begin{array}{c}\text { TFBS \# } \\
\text { (in TSS } \pm 500 \mathrm{bp} \text { ) }\end{array}$} & \multicolumn{2}{|c|}{$\begin{array}{l}\text { Peak \# of TFBS } \\
\text { (in TSS } \pm 500 \mathrm{bp} \text { ) }\end{array}$} & \multicolumn{2}{|c|}{$\begin{array}{l}\% \text { of TFBS Peak } \\
\text { (in TSS } \pm 500 \text { bp) }\end{array}$} & \multicolumn{2}{|c|}{ Gene \# } \\
\hline & & & & $\mathrm{Oh}$ & $12 \mathrm{~h}$ & $\mathrm{Oh}$ & $12 \mathrm{~h}$ & $\mathrm{Oh}$ & $12 \mathrm{~h}$ \\
\hline 1 & ETS1 & V\$CETS1P54_01 & 1587 & 341 & 262 & $21.5 \%$ & $16.5 \%$ & 294 & 229 \\
\hline 2 & GABPA/NRF-2 & V\$NRF2_01 & 1577 & 336 & 237 & $21.3 \%$ & $15.0 \%$ & 286 & 206 \\
\hline 3 & ELK1 & V\$ELK1_02 & 1667 & 333 & 254 & $20.0 \%$ & $15.2 \%$ & 279 & 215 \\
\hline \multirow[t]{2}{*}{4} & USF1 & V\$USF_C & 441 & 79 & 45 & $17.9 \%$ & $10.2 \%$ & 71 & 44 \\
\hline & & V\$USF_Q6 & 1136 & 146 & 97 & $12.9 \%$ & $8.5 \%$ & 132 & 92 \\
\hline \multirow[t]{2}{*}{5} & STAT1 & V\$STAT1_03 & 282 & 50 & 41 & $17.7 \%$ & $14.5 \%$ & 48 & 39 \\
\hline & & V\$STAT1_01 & 1179 & 146 & 122 & $12.4 \%$ & $10.3 \%$ & 134 & 110 \\
\hline 6 & STAT4 & V\$STAT4_01 & 106 & 17 & 17 & $16.0 \%$ & $16.0 \%$ & 4 & 4 \\
\hline \multirow[t]{2}{*}{7} & MYC/MAX & V\$MYCMAX_02 & 580 & 90 & 76 & $15.5 \%$ & $13.1 \%$ & 68 & 55 \\
\hline & & V\$MYCMAX_01 & 784 & 104 & 88 & $13.3 \%$ & $11.2 \%$ & 93 & 68 \\
\hline 8 & CREB1 & V\$CREB_01 & 666 & 103 & 87 & $15.5 \%$ & $13.1 \%$ & 97 & 83 \\
\hline 9 & MAX & V\$MAX_01 & 584 & 87 & 68 & $14.9 \%$ & $11.6 \%$ & 75 & 64 \\
\hline 10 & ATF-2 & V\$CREBP1_01 & 272 & 37 & 30 & $13.6 \%$ & $11.0 \%$ & 35 & 28 \\
\hline 11 & STAT3 & V\$STAT3_01 & 986 & 133 & 108 & $13.5 \%$ & $11.0 \%$ & 123 & 101 \\
\hline 12 & SREBF1/SREBP1 & V\$SREBP1_01 & 1038 & 140 & 108 & $13.5 \%$ & $10.4 \%$ & 110 & 93 \\
\hline 13 & MYCN & V\$NMYC_01 & 1467 & 195 & 125 & $13.3 \%$ & $8.5 \%$ & 170 & 114 \\
\hline 14 & STAT6 & V\$STAT_01 & 570 & 75 & 59 & $13.2 \%$ & $10.4 \%$ & 66 & 54 \\
\hline 15 & ARNT & V\$ARNT_01 & 835 & 108 & 89 & $12.9 \%$ & $10.7 \%$ & 102 & 87 \\
\hline 16 & ARID5B/MRF2 & V\$MRF2_01 & 478 & 61 & 57 & $12.8 \%$ & $11.9 \%$ & 56 & 52 \\
\hline 17 & E2F1/2/3/4/5 & V\$E2F_02 & 1301 & 166 & 121 & $12.8 \%$ & $9.3 \%$ & 149 & 110 \\
\hline 18 & PBX1 & V\$PBX1_01 & 220 & 28 & 21 & $12.7 \%$ & $9.5 \%$ & 28 & 21 \\
\hline
\end{tabular}

bold: transcription factor with evidence of SUMO modification.

Interestingly, we found that there were three IRFs, IRF-7, IRF-1, and IRF-2 that do not exist in the SUMO$2 / 3$ target TFs list before viral reactivation, but are now listed as the 4th, 5th and 6th top-most TFs, respectively, after viral reactivation. Comparing the SUMO tagging of these IRFs before and after viral reactivation, we found that all three IRFs binding sites are preferentially subjected to SUMO-2/3 modification after viral reactivation (Figure 6). To confirm that SUMO-2/3 enrichment at the IRF-1, IRF-2 and IRF-7 binding sites occurs during viral reactivation, we design primers targeting the IRFs binding regions where the SUMO-2/3 peaks had been identified by the ChIP-seq assay. The SUMO-2/3 enrichment in those regions was then validated using a ChIP sample and real-time quantitative-PCR (qPCR). Consistent with the ChIP-seq results, the 12 IRF binding regions tested here showed significant enrichment after viral reactivation for SUMO-2/3 but not for SUMO-1 compare to the non-reactivated control cells (Figure 7). ChIP-reChIP analyses further confirmed the colocalization of IRF-7 and SUMO-2/3 on IRF-7 binding region with SUMO-2/3 enrichment (Figure 8).
The identification of IRF-1, IRF-2 and IRF-7 as potential SUMO-2/3 targets during KSHV reactivation suggests that the viral SUMO E3 ligase K-bZIP may be involved in this phenomenon. To address this, we first cloned all three IRFs using CDNA of BCBL-1 cells. Then $293 \mathrm{~T}$ cells were transiently co-transfected with Flagtagged IRF-1, IRF-2 or IRF-7, T7-tagged SUMO-2 and SUMO-3, and HA-tagged K-bZIP; this was followed by immunoblotting or immunoprecipitation using Flag antibody. The results showed an increase in SUMO modification of IRF-1 and IRF-2 when there was overexpression of K-bZIP (Figure right panel of 9A and B). SUMOylation of IRF-1 and IRF-2 was further confirmed by immunoblotting using anti-SUMO-2/3 antibody (Figure left panel of 9A and B). Although we were unable to identify SUMOylation of IRF-7 using this approach (data not shown), an immunoprecipitation assay showed that IRF-7 is able to interact with K-bZIP (Figure 9C and D). Thus SUMO-mediated transcription regulation not only involves covalent SUMO modification of transcription regulatory proteins, but also seems to involve SUMO modified co-regulatory proteins that 
Table 2 Top 20 potential SUMO-2/3 targeting TFs before KSHV reactivation

\begin{tabular}{|c|c|c|c|c|c|c|c|c|c|}
\hline \multirow[t]{2}{*}{ Rank } & \multirow[t]{2}{*}{ Name } & \multirow[t]{2}{*}{ Transfac } & \multirow[t]{2}{*}{$\begin{array}{c}\text { TFBS \# } \\
\text { (in TSS } \pm 500 \mathrm{bp} \text { ) }\end{array}$} & \multicolumn{2}{|c|}{$\begin{array}{l}\text { Peak \# of TFBS } \\
\text { (in TSS } \pm 500 \text { bp) }\end{array}$} & \multicolumn{2}{|c|}{$\begin{array}{l}\% \text { of TFBS Peak } \\
\text { (in TSS } \pm 500 \text { bp) }\end{array}$} & \multicolumn{2}{|c|}{ Gene \# } \\
\hline & & & & $\mathrm{Oh}$ & $12 \mathrm{~h}$ & $\mathrm{Oh}$ & $12 \mathrm{~h}$ & $\overline{O h}$ & $12 \mathrm{~h}$ \\
\hline 1 & ETS1 & V\$CETS1P54_01 & 1587 & 337 & 397 & $21.2 \%$ & $25.0 \%$ & 296 & 353 \\
\hline 2 & GABPA/NRF-2 & V\$NRF2_01 & 1577 & 320 & 379 & $20.3 \%$ & $24.0 \%$ & 274 & 335 \\
\hline 3 & ELK1 & V\$ELK1_02 & 1667 & 336 & 382 & $20.2 \%$ & $22.9 \%$ & 285 & 328 \\
\hline 4 & STAT4 & V\$STAT4_01 & 106 & 20 & 12 & $18.9 \%$ & $11.3 \%$ & 7 & 12 \\
\hline 5 & STAT1 & V\$STAT1_03 & 282 & 53 & 79 & $18.8 \%$ & $28.0 \%$ & 45 & 68 \\
\hline 6 & CREB1 & V\$CREB_01 & 666 & 124 & 164 & $18.6 \%$ & $24.6 \%$ & 114 & 152 \\
\hline \multirow[t]{2}{*}{7} & ATF2 & V\$CREBP1_01 & 272 & 45 & 67 & $16.5 \%$ & $24.6 \%$ & 45 & 64 \\
\hline & & V\$CREBP1_Q2 & 1273 & 168 & 201 & $13.2 \%$ & $15.8 \%$ & 135 & 198 \\
\hline 8 & STAT6 & V\$STAT_01 & 570 & 92 & 122 & $16.1 \%$ & $21.4 \%$ & 85 & 110 \\
\hline 9 & MYC/MAX & V\$MYCMAX_02 & 580 & 93 & 96 & $16.0 \%$ & $16.6 \%$ & 74 & 87 \\
\hline 10 & MAX & V\$MAX_01 & 584 & 93 & 110 & $15.9 \%$ & $18.8 \%$ & 79 & 97 \\
\hline 11 & HLF & V\$HLF_01 & 438 & 69 & 90 & $15.8 \%$ & $20.5 \%$ & 66 & 85 \\
\hline 12 & USF1 & V\$USF_C & 441 & 69 & 86 & $15.6 \%$ & $19.5 \%$ & 64 & 78 \\
\hline 13 & ARID5B/MRF2 & V\$MRF2_01 & 478 & 74 & 100 & $15.5 \%$ & $20.9 \%$ & 68 & 91 \\
\hline \multirow[t]{2}{*}{14} & NFYA/B/C & V\$NFY_Q6 & 1557 & 217 & 295 & $13.9 \%$ & $18.9 \%$ & 176 & 250 \\
\hline & & V\$NFY_C & 416 & 57 & 79 & $13.7 \%$ & $19.0 \%$ & 55 & 75 \\
\hline 15 & SREBF1/SREBP 1 & V\$SREBP1_01 & 1038 & 144 & 179 & $13.9 \%$ & $17.2 \%$ & 100 & 170 \\
\hline 16 & STAT5A & V\$STAT5A_02 & 982 & 135 & 155 & $13.7 \%$ & $15.8 \%$ & 109 & 133 \\
\hline 17 & ZEB1 & V\$AREB6_04 & 530 & 70 & 89 & $13.2 \%$ & $16.8 \%$ & 60 & 78 \\
\hline 18 & ARNT & V\$ARNT_01 & 835 & 110 & 136 & $13.2 \%$ & $16.3 \%$ & 106 & 131 \\
\hline 19 & $\mathrm{E} 2 \mathrm{~F} 1 / 2 / 3 / 4 / 5$ & V\$E2F_02 & 1301 & 171 & 226 & $13.1 \%$ & $17.4 \%$ & 155 & 199 \\
\hline 20 & STAT3 & V\$STAT3_01 & 986 & 129 & 168 & $13.1 \%$ & $17.0 \%$ & 127 & 159 \\
\hline
\end{tabular}

show a non-covalent association at the TFBS. These findings suggest that IRF-7 may recruit K-bZIP to its binding sites together with other K-bZIP SUMOylated chromatin binding protein(s); these are then able to be co-immunoprecipitated (co-IPed) by SUMO antibody.
SUMO-2/3 is enriched on promoters of immune-related genes that are unaltered during KSHV reactivation

To study the functional role of SUMO-2/3 in the regulation of gene expression during KSHV reactivation, we conducted a detailed RNA-seq analysis using TREx-

Table 3 Top 20 potential SUMO-1 targeting TFs after KSHV reactivation

\begin{tabular}{|c|c|c|c|c|c|c|c|c|c|}
\hline \multirow[t]{2}{*}{ Rank } & \multirow[t]{2}{*}{ Name } & \multirow[t]{2}{*}{ Transfac } & \multirow[t]{2}{*}{$\begin{array}{c}\text { TFBS \# } \\
\text { (in TSS } \pm 500 \mathrm{bp} \text { ) }\end{array}$} & \multicolumn{2}{|c|}{$\begin{array}{l}\text { Peak \# of TFBS } \\
\text { (in TSS } \pm 500 \text { bp) }\end{array}$} & \multicolumn{2}{|c|}{$\begin{array}{l}\% \text { of TFBS Peak } \\
\text { (in TSS } \pm 500 \text { bp) }\end{array}$} & \multicolumn{2}{|c|}{ Gene \# } \\
\hline & & & & $\mathrm{Oh}$ & $12 \mathrm{~h}$ & $\mathrm{Oh}$ & $12 \mathrm{~h}$ & $\overline{\mathrm{Oh}}$ & $\overline{12 \mathrm{~h}}$ \\
\hline 1 & ETS1 & V\$CETS1P54_01 & 1587 & 341 & 262 & $21.5 \%$ & $16.5 \%$ & 294 & 229 \\
\hline 2 & STAT4 & V\$STAT4_01 & 106 & 17 & 17 & $16.0 \%$ & $16.0 \%$ & 4 & 4 \\
\hline 3 & ELK1 & V\$ELK1_02 & 1667 & 333 & 254 & $20.0 \%$ & $15.2 \%$ & 279 & 215 \\
\hline 4 & GABPA/NRF-2 & V\$NRF2_01 & 1577 & 336 & 237 & $21.3 \%$ & $15.0 \%$ & 286 & 206 \\
\hline 5 & STAT1 & V\$STAT1_03 & 282 & 50 & 41 & $17.7 \%$ & $14.5 \%$ & 48 & 39 \\
\hline 6 & NFATC1/2/3/4 & V\$NFAT_Q6 & 487 & 48 & 70 & $9.9 \%$ & $14.4 \%$ & 22 & 23 \\
\hline 7 & STAT5A & V\$STAT5A_02 & 982 & 118 & 129 & $12.0 \%$ & $13.1 \%$ & 95 & 104 \\
\hline 8 & MYC/MAX & V\$MYCMAX_02 & 580 & 90 & 76 & $15.5 \%$ & $13.1 \%$ & 68 & 55 \\
\hline 9 & CREB1 & V\$CREB_01 & 666 & 103 & 87 & $15.5 \%$ & $13.1 \%$ & 97 & 83 \\
\hline 10 & CUTL1 & V\$CDPCR1_01 & 582 & 59 & 73 & $10.1 \%$ & $12.5 \%$ & 54 & 49 \\
\hline
\end{tabular}


Table 4 Top 20 potential SUMO-2/3 targeting TFs after KSHV reactivation

\begin{tabular}{|c|c|c|c|c|c|c|c|c|c|}
\hline \multirow[t]{2}{*}{ Rank } & \multirow[t]{2}{*}{ Name } & \multirow[t]{2}{*}{ Transfac } & \multirow[t]{2}{*}{$\begin{array}{c}\text { TFBS \# } \\
\text { (in TSS } \pm 500 \mathrm{bp} \text { ) }\end{array}$} & \multicolumn{2}{|c|}{$\begin{array}{l}\text { Peak \# of TFBS } \\
\text { (in TSS } \pm 500 \mathrm{bp} \text { ) }\end{array}$} & \multicolumn{2}{|c|}{$\begin{array}{l}\% \text { of TFBS Peak } \\
\text { (in TSS } \pm 500 \mathrm{bp} \text { ) }\end{array}$} & \multicolumn{2}{|c|}{ Gene \# } \\
\hline & & & & $\mathrm{Oh}$ & $12 \mathrm{~h}$ & $\mathrm{Oh}$ & $12 \mathrm{~h}$ & $\mathrm{Oh}$ & $12 \mathrm{~h}$ \\
\hline 1 & STAT1 & V\$STAT1_03 & 282 & 53 & 79 & $18.8 \%$ & $28.0 \%$ & 45 & 68 \\
\hline 2 & ETS1 & V\$CETS1P54_01 & 1587 & 337 & 397 & $21.2 \%$ & $25.0 \%$ & 296 & 353 \\
\hline 3 & PBX1 & V\$PBX1_01 & 220 & 28 & 55 & $12.7 \%$ & $25.0 \%$ & 28 & 54 \\
\hline 4 & ATF2 & V\$CREBP1_01 & 272 & 45 & 67 & $16.5 \%$ & $24.6 \%$ & 45 & 64 \\
\hline 5 & CREB1 & V\$CREB_01 & 666 & 124 & 164 & $18.6 \%$ & $24.6 \%$ & 114 & 152 \\
\hline 6 & GABPA/NRF-2 & V\$NRF2_01 & 1577 & 320 & 379 & $20.3 \%$ & $24.0 \%$ & 274 & 335 \\
\hline 7 & ELK1 & V\$ELK1_02 & 1667 & 336 & 382 & $20.2 \%$ & $22.9 \%$ & 285 & 328 \\
\hline 8 & IRF-1 & V\$IRF1_01 & 534 & 46 & 118 & $8.6 \%$ & $22.1 \%$ & 43 & 113 \\
\hline 9 & STAT6 & V\$STAT_01 & 570 & 92 & 122 & $16.1 \%$ & $21.4 \%$ & 85 & 110 \\
\hline 10 & FOXO4 & V\$FOXO4_02 & 456 & 28 & 97 & $6.1 \%$ & $21.3 \%$ & 28 & 75 \\
\hline 11 & NFATC1/2/3/4 & V\$NFAT_Q6 & 487 & 50 & 102 & $10.3 \%$ & $20.9 \%$ & 29 & 55 \\
\hline 12 & ARID5B/MRF2 & V\$MRF2_01 & 478 & 74 & 100 & $15.5 \%$ & $20.9 \%$ & 68 & 91 \\
\hline 13 & IRF-2 & V\$IRF2_01 & 601 & 48 & 125 & $8.0 \%$ & $20.8 \%$ & 63 & 93 \\
\hline 14 & SRY & V\$SRY_01 & 523 & 32 & 108 & $6.1 \%$ & $20.7 \%$ & 30 & 91 \\
\hline 15 & HLF & V\$HLF_01 & 438 & 69 & 90 & $15.8 \%$ & $20.5 \%$ & 66 & 85 \\
\hline 16 & IRF-7 & V\$IRF7_01 & 754 & 87 & 153 & $11.5 \%$ & $20.3 \%$ & 79 & 126 \\
\hline 17 & MEF2A & V\$MEF2_01 & 455 & 36 & 91 & $7.9 \%$ & $20.0 \%$ & 28 & 72 \\
\hline 18 & GATA1 & V\$GATA1_04 & 191 & 14 & 38 & $7.3 \%$ & $19.9 \%$ & 13 & 35 \\
\hline 19 & POU3F2 & V\$POU3F2_02 & 246 & 17 & 48 & $6.9 \%$ & $19.5 \%$ & 17 & 43 \\
\hline 20 & USF1 & V\$USF_C & 441 & 69 & 86 & $15.6 \%$ & $19.5 \%$ & 64 & 78 \\
\hline
\end{tabular}

bold: transcription factor with evidence of SUMO modification.

F3H3-K-Rta BCBL-1 cells before and after K-Rta induction for viral reactivation. We sorted 26008 genes using expression levels based on FPKM into five groups, namely no expression (FPKM <0.05: 7954), low expression (FPKM $0.05 \sim<1:$ 6503), medium expression (FPKM $1 \sim<10$ : 5597), high expression (FPKM $10 \sim<100: 5390$ ), and very high expression (FPKM >100: 564). We found that between $27 \%$ and $37 \%$ of the very high and high expression group, about $16 \%$ of the medium expression group, about $4 \%$ of the low expression group, and about $1 \%$ of the no expression group promoters were labeled by SUMO-1 or SUMO-2/3 (Figure 10A). Consistent with a previous study using yeast [7] and a study of SUMO-1 using HeLa cells [46], both of which showed that SUMO preferentially occupies transcriptionally active genes, the modification by all SUMO paralogues explored here also seems to be present at greater levels on the promoters of genes that show a higher level of expression. Twenty-four hours after KSHV reactivation, we found a significant increase in SUMO$2 / 3$ binding at the promoters of genes with high expression $(\sim 15 \%)$ and medium expression $(\sim 10 \%)$. This compared with little SUMO-2/3 binding enrichment at the promoters of genes with low expression $(\sim 2 \%)$ and no expression $(<1 \%)$ (Figure 10C). In contrast, there was a slight decrease in SUMO-1 modification across all expression categories (Figure 10B). These results indicate that SUMO-1 and SUMO-2/3 modifications are important for maintaining the transcription profiles of the non-reactivated control cells. Nevertheless, the increase in SUMO-2/3 modification during KSHV reactivation supports the notion that specific SUMO-2/3 targeting is important to transcription regulation during the KSHV life cycle.

In addition to maintaining constitutive transcription, SUMO has also been found to prevents the overt activation of induced genes by facilitating the shut off of the transcription in yeast [7]. To assess the effect of SUMO$2 / 3$ enrichment on the shut-off of transcription, we compared global host gene expression in BCBL-1 cells before and after KSHV reactivation. We found that among the $\sim 18,000$ transcriptionally active host genes (genes with FPKM $>0.05$ ), only $\sim 2,600$ of the up-regulated genes and $\sim 2,200$ of the down-regulated genes were changed more than 1.5-fold in response to KSHV reactivation for 24 hours. A similar result was found at 12 hours after viral 


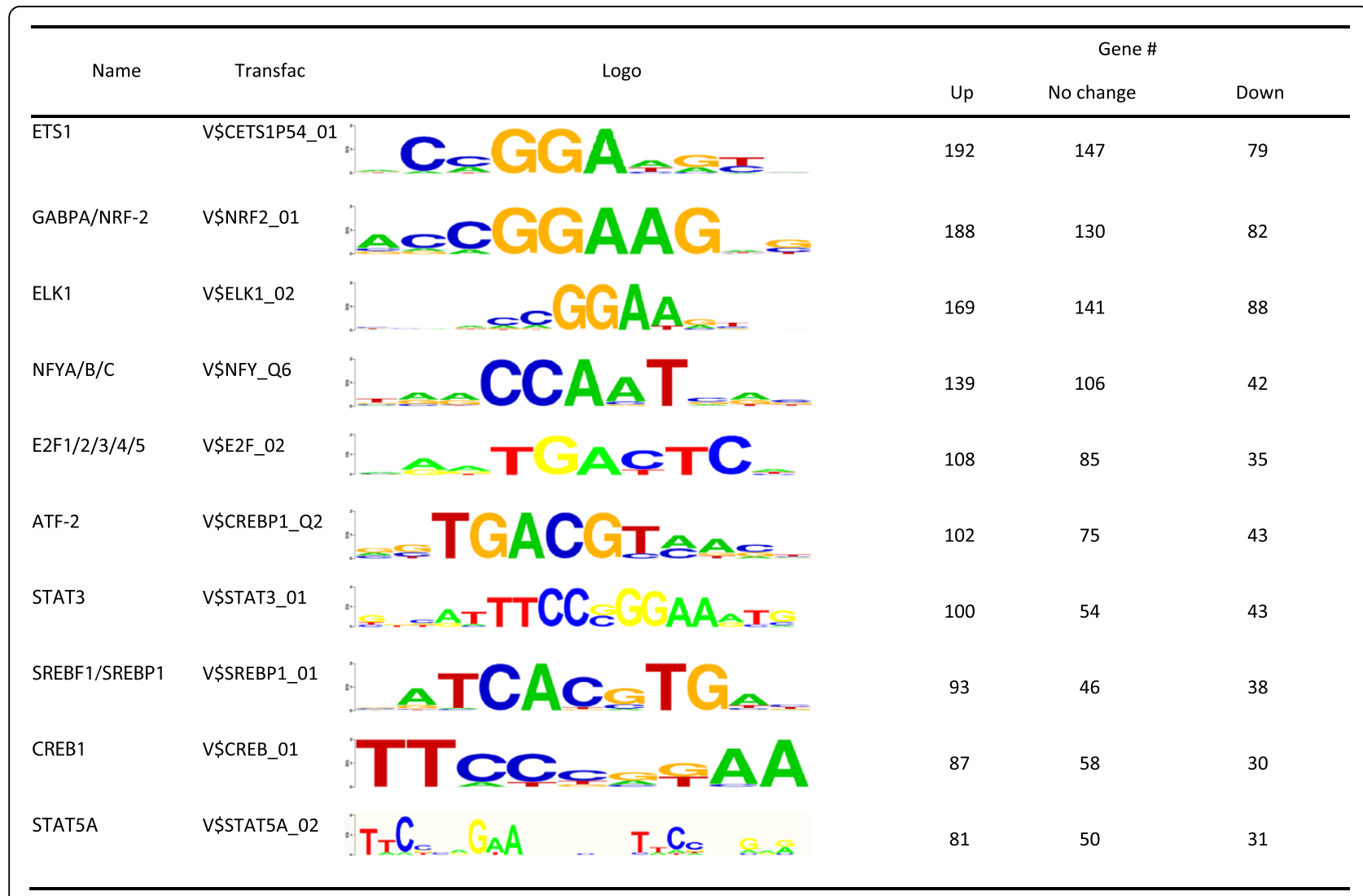

Figure 4 Top 10 potential SUMO-2/3 targeting TFs ranking by gene number before KSHV reactivation. bold: transcription factor with evidence of SUMO modification.

reactivation $(\sim 1,900$ up-regulated and $\sim 2,100$ downregulated genes). Analysis of the SUMO peak distributions within the promoter region of these transcriptionally upregulated, down-regulated, and no change genes showed that the predominant association is between SUMO enrichment peaks and genes that can be shown to exhibit no change in expression. After viral reactivation for 24 hours, there was a significant increase in SUMO-2/3 recruitment to the promoters of transcriptionally unaltered genes (Figure 11A and B). When we further grouped the SUMO peaks into increased binding, decrease binding and no change in binding during viral reactivation, a similar result was found (Figure $11 \mathrm{C}$ to E). SUMO-2/3 peak enrichment during viral reactivation was predominantly associated with transcriptionally unaltered genes. When we analyzed the association of the expression level of the transcriptionally up-regulated, down-regulated and unaltered genes with SUMO peaks during viral reactivation, we found that most of the viral up-regulated $(>80 \%)$ and down-regulated $(>90 \%)$ genes fall into the low (FPKM $0.05 \sim 1$ ) and no expression (FPKM <0.05) gene categories that show little SUMO-2/3 modification on their promoter (Figure $12 \mathrm{~A}$ and $\mathrm{B}$ ).
Furthermore and interestingly, more than $75 \%$ of the expression-unchanged genes, which contain a higher proportion of SUMO-2/3 modification on their promoter, are in the medium and high expression gene categories (Figure $12 \mathrm{C}$ and Figure 13). This result indicates that SUMO-2/3 enrichment during viral reactivation may contribute to "stabilizing" the transcriptional activity of these medium (FPKM $1 \sim 10)$ and high (FPKM >10) expression genes during viral reactivation. To confirm the RNA-seq data, we design primers for IRF-1, IRF-2 and IRF-7 targeted and transcriptionally active genes that show no change in expression during viral reactivation in BCBL-1 cells. The lack of change in expression during viral reactivation was confirmed using cDNA samples and real-time qPCR. Consistent with the RNA-seq results, the 12 genes tested here showed no changes in expression level compared to the control after K-Rta-induced KSHV reactivation (Figure 14). To further study the "stabilizing" potential of SUMO-2/3 in transcription regulation, we generated an inducible SUMO-2/3 knockdown BCBL-1 cell line, TREx-F3H3-KRta-shSUMO-2/3 BCBL-1. Western blot analysis shows the successful knockdown of SUMO-2/3 at 24 and 48 


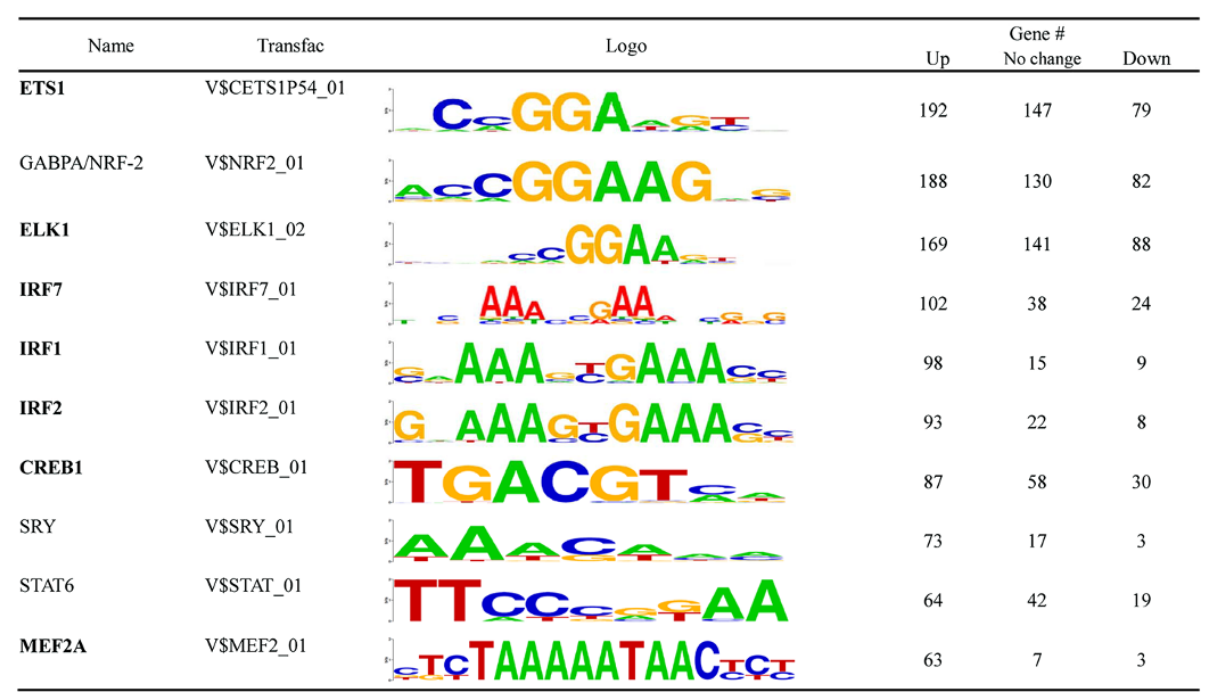

Figure 5 Top 10 potential SUMO-2/3 targeting TFs ranking by gene number after KSHV reactivation. bold: transcription factor with evidence of SUMO modification.

hours after induction (Figure 14A). Consistent with our hypothesis, qPCR analysis showed a higher induction of most of the 12 genes we analyzed after SUMO-2/3 knockdown during viral reactivation (Figure 14B). Again, these results imply that SUMO-2/3 enrichment within the host promoter region during KSHV reactivation is closely related to preventing transcriptional activation of constitutively active host genes, many of which are immune response genes (see below).

To determine whether SUMO-2/3 target a group of genes with specific functions, we carried out a gene ontology (GO) analysis of genes that are targeted by SUMO- 1 and SUMO-2/3 before and after viral reactivation using the IPA software. We found that the genes targeted by SUMO-2/3 after viral reactivation are significantly involved in several pathways related to cellular immune responses, cytokine signaling, cell growth, apoptosis and cancer (Table 5). We further analyzed SUMO targeted genes with no change in transcription and genes with no change in transcription but increased in SUMO-2/3 enrichment. Consistently, we found that the transcriptionally unaltered genes targeted by SUMO$2 / 3$ after viral reactivation are significantly involved in cellular immune responses (Table 6). Taken together, all of the present results support the notion that KSHV may target SUMO-2/3 modified proteins to active chromatin regions to prevent overt activation of various important genes during viral reactivation, especially those involved in the innate immune response.

\section{Discussion and conclusions}

SUMO is a multifaceted modifier of chromatin structure. SUMO modification of chromatin proteins regulates a range of cellular processes including transcription, repli-

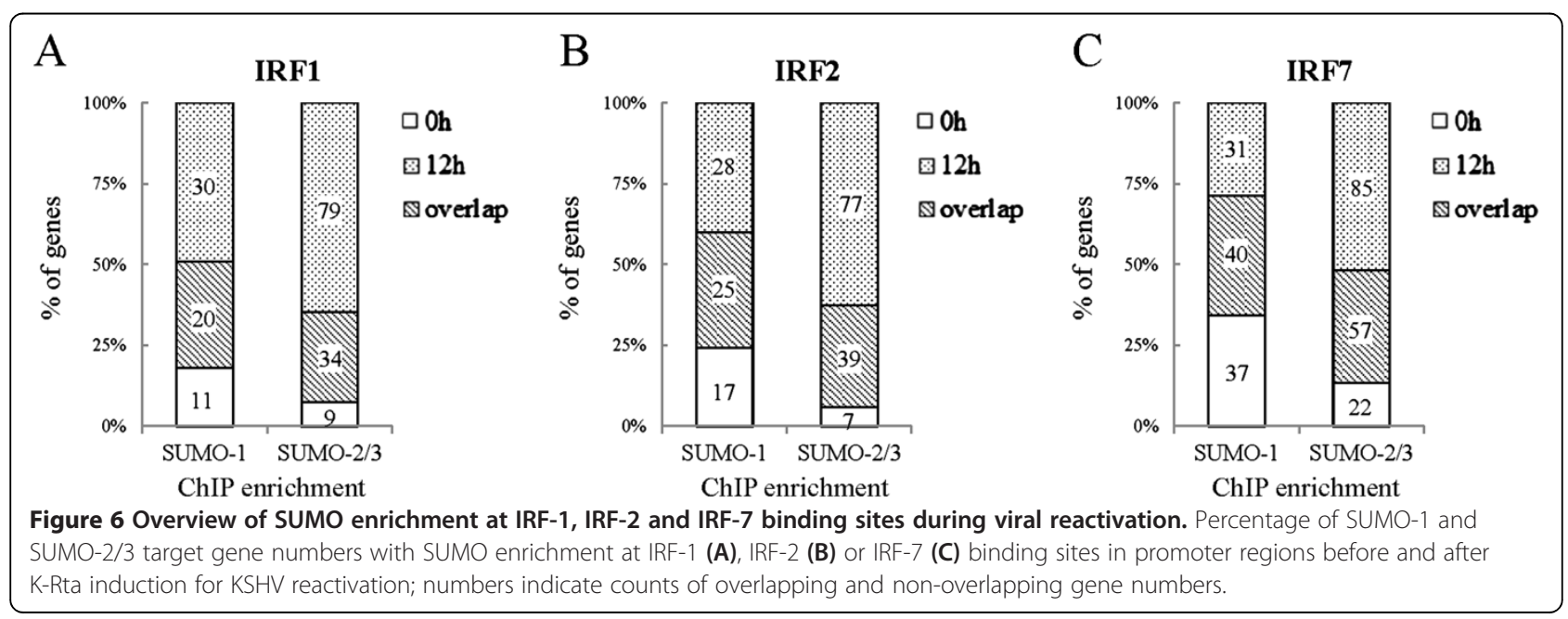




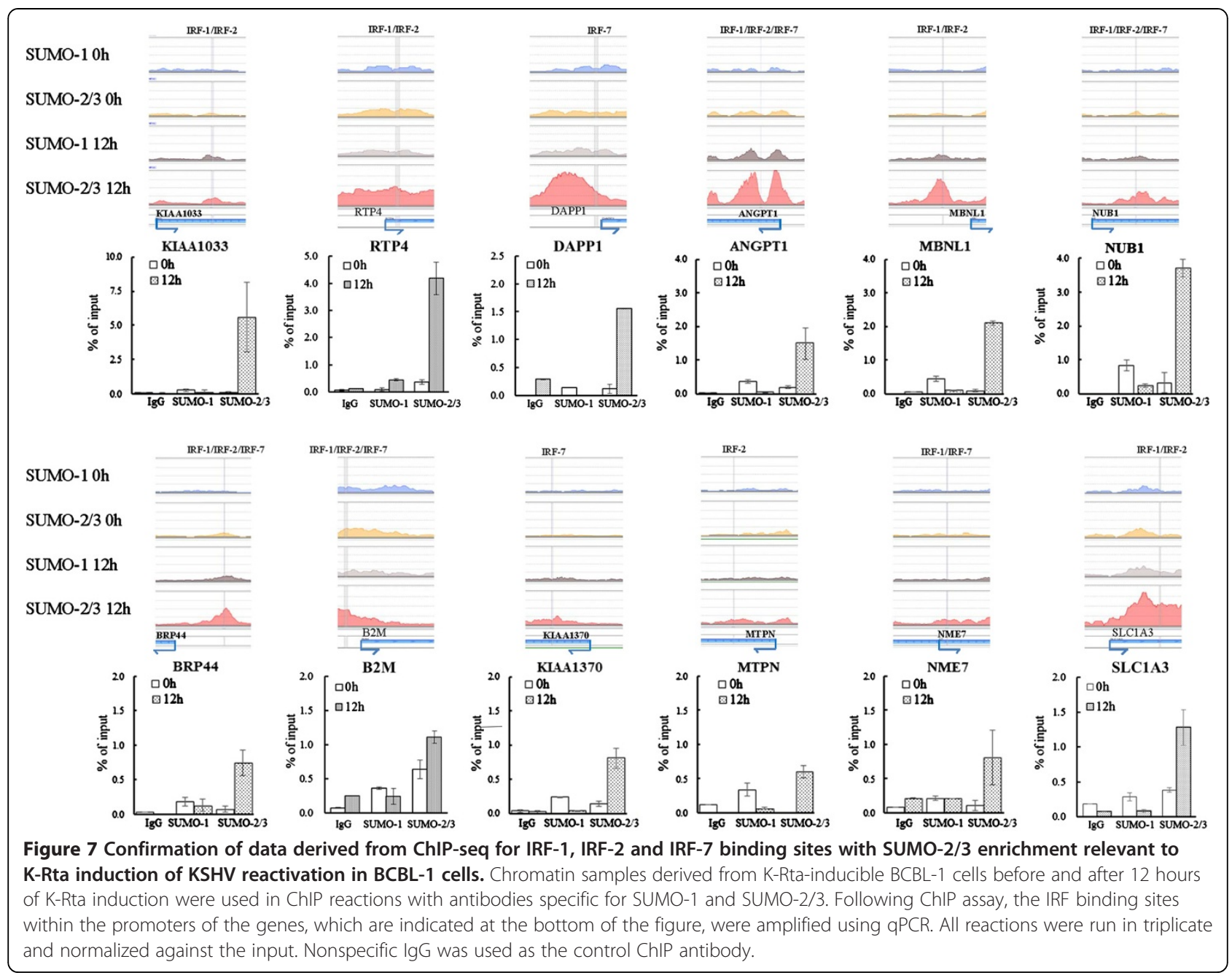

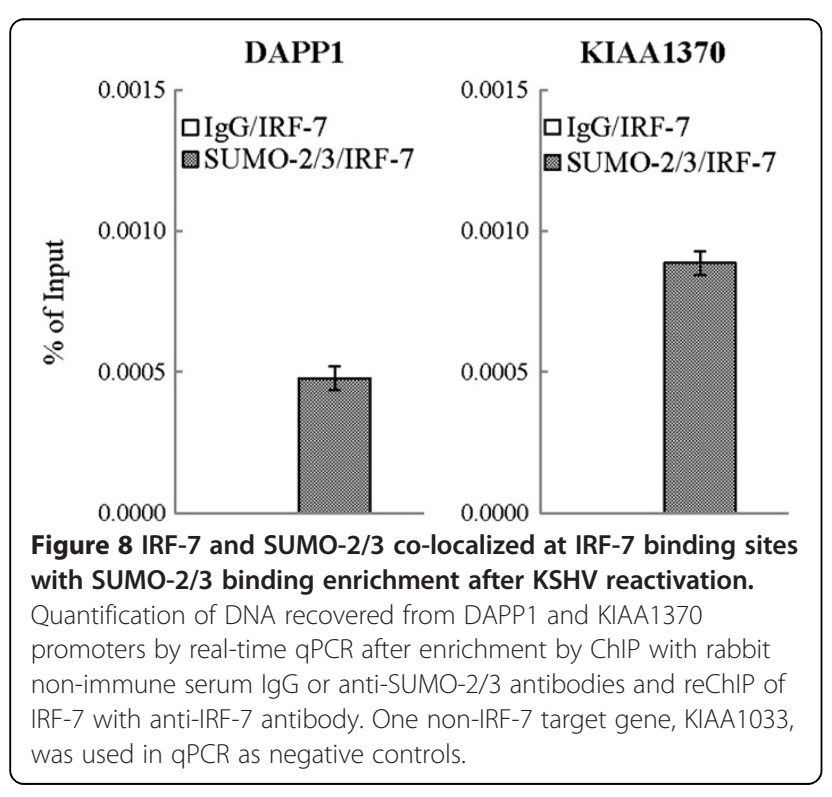

cation, DNA repair and chromosome segregation. SUMOylation has long been believe to be associated with gene silencing or repression. However, global mapping of chromatin binding by SUMO in yeast [7] and Drosophila [50], show that SUMOylated proteins are present at transcriptionally active and induced genes. This discovery led to the hypothesis that SUMO functions to prevent superinduction of actively transcribed genes by external factors (in this case, viral infection) to maintain a steady-state level of transcription. However, lower eukaryotes possess only one SUMO isoform, whereas there are two groups of SUMO variants in humans; SUMO-1 and SUMO-2/3. Recently, the global chromatin localization of SUMO-1 through the cell cycle of human HeLa cells has been identified. Similar to that reported in yeast, SUMO-1 tends to cluster around transcriptionally active genes [46]. Although increasing evidence from studies targeting specific cellular factors suggests that there is differential conjugation and functionality among SUMO paralogues, the global functional heterogeneity of human SUMO paralogues seems to be limited in their conjugation dynamics $[11,12]$ 


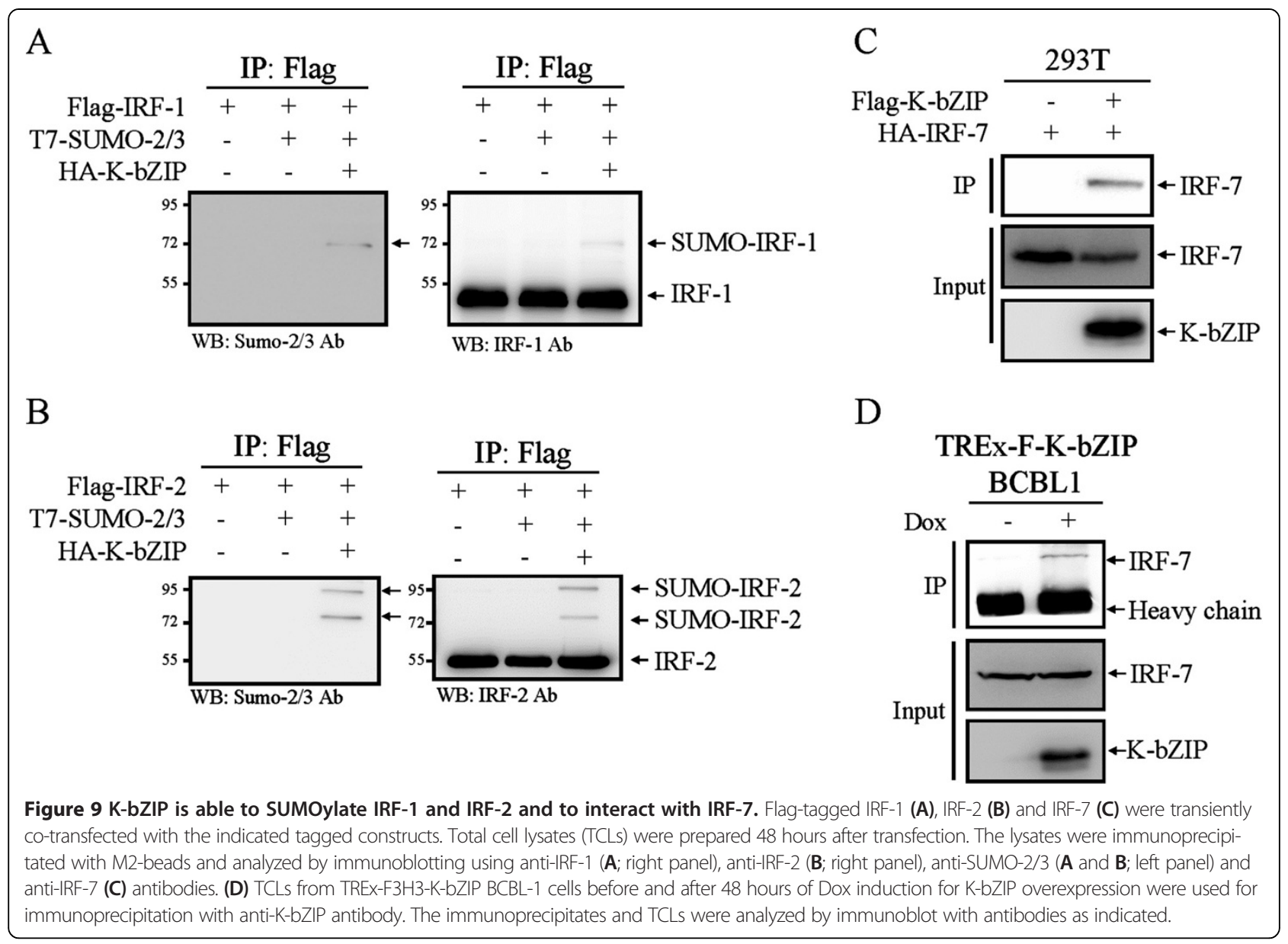

and subcellular localizations [51]. The global functional differences between SUMO paralogues in terms of epigenetic regulation remains a puzzle. In this study, we compared the chromosome-wide labeling of SUMO-1 and SUMO-2/3 proteins before and after herpesvirus reactivation using the ChIP-Seq assay. We found that firstly, on a genome-wide scale, the binding profile of the SUMO paralogues was highly similar in the control cells, but that differences were evident after KSHV reactivation with there being a significant increase in SUMO-2/3 binding while there was only limited changes in the SUMO-1 binding profile. Secondly, the distribution of both SUMO paralogues on the chromatin showed a greater tendency toward being associated with transcription regulatory

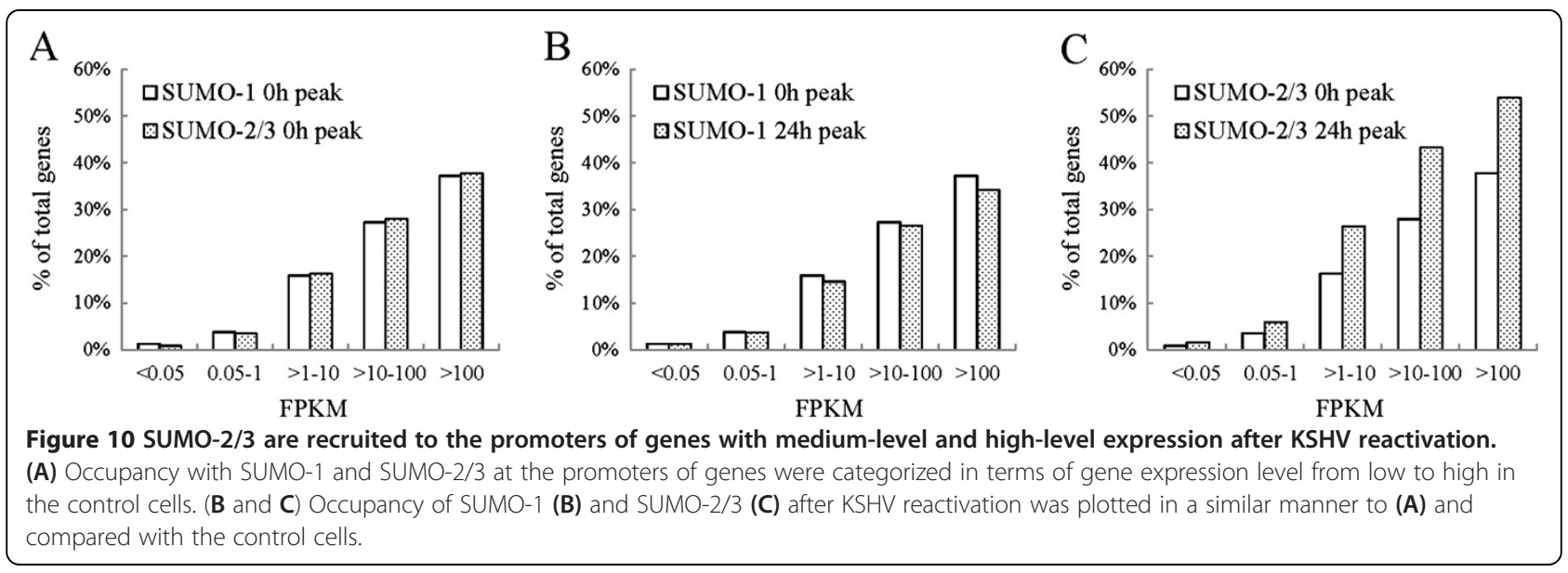




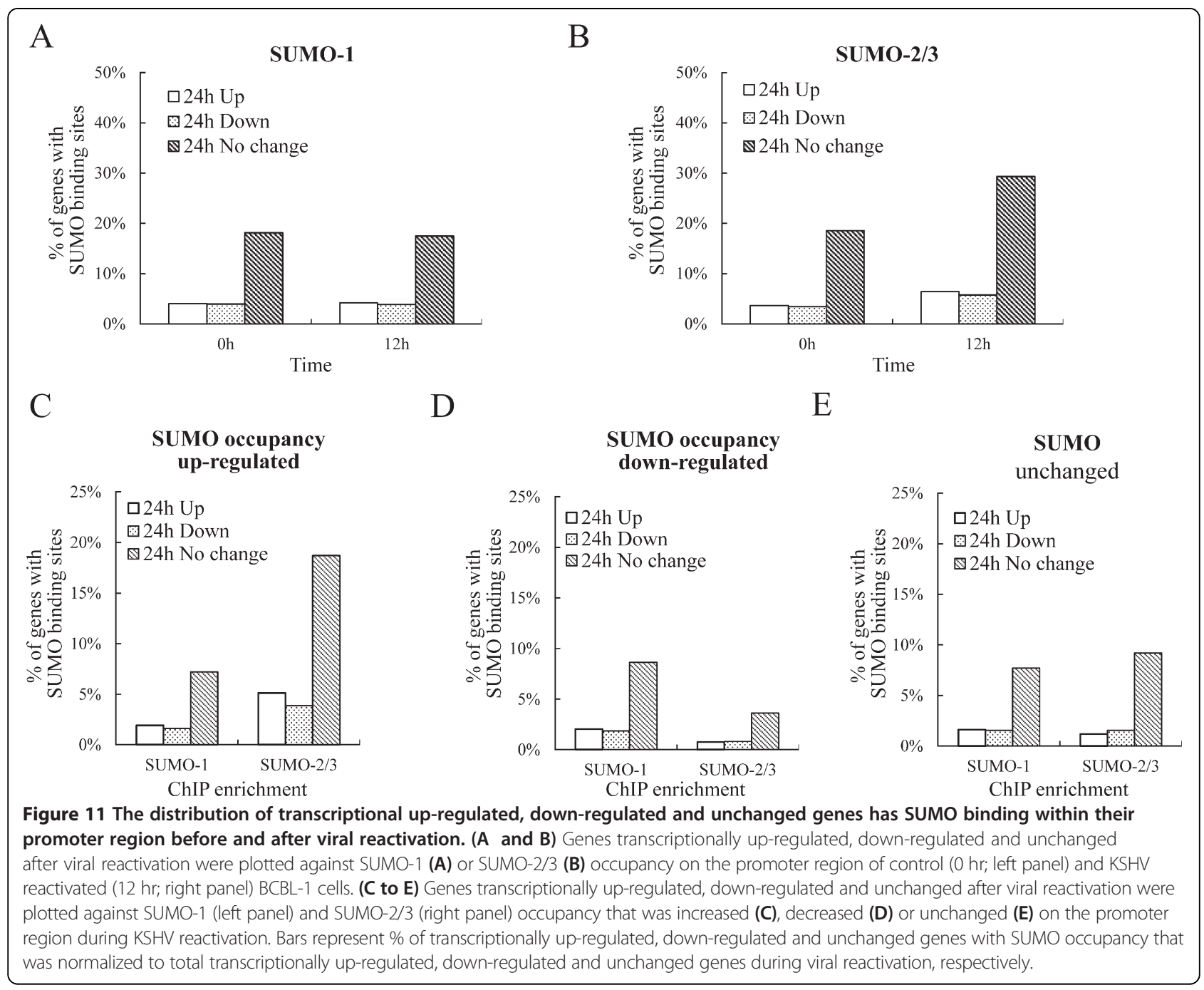

regions (promoters) and that, furthermore, the binding of SUMO-2/3 onto the promoter regions was significantly increased during viral reactivation. Thirdly, there was a dramatic increase in SUMO-2/3 binding and a slight decrease in SUMO-1 binding onto TFBSs during viral reactivation. Fourthly, the potential SUMO-1 and SUMO-2/3 target TFs highly overlapped in the control cells, while the SUMO-2/3 specific TFs are significantly increased during viral reactivation. Fifthly, three IRFs, "the master regulators of immune responses" show up in the top-10 most important gene-regulating TFs targeted by SUMO-2/3 after KSHV reactivation. Sixth, both the SUMO paralogues

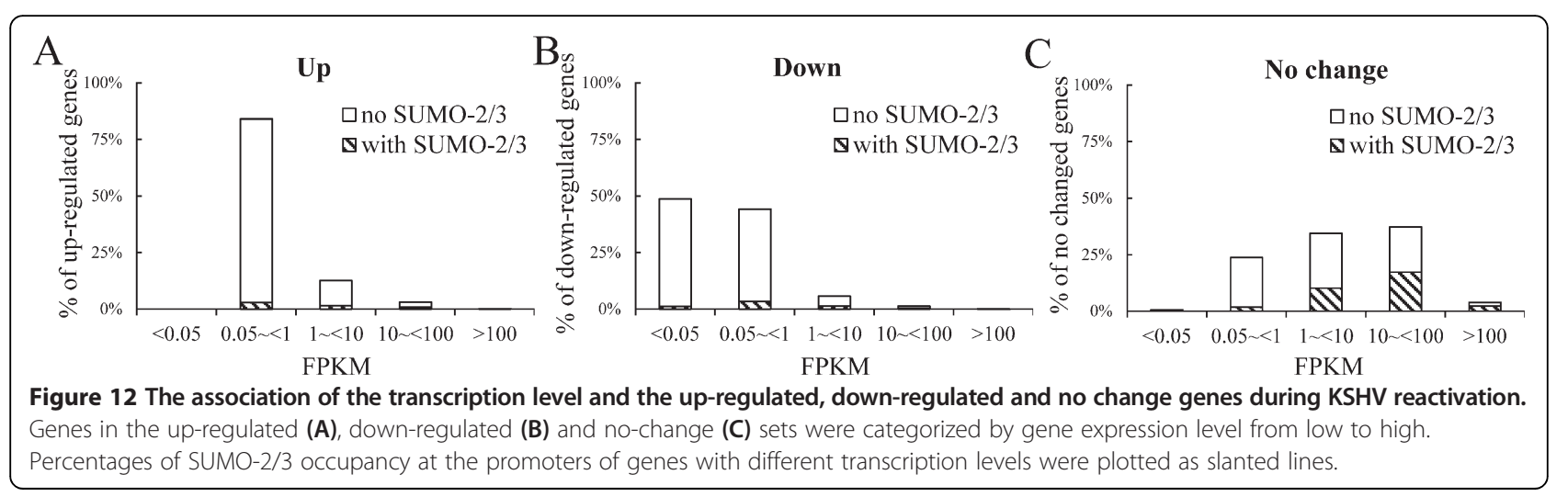




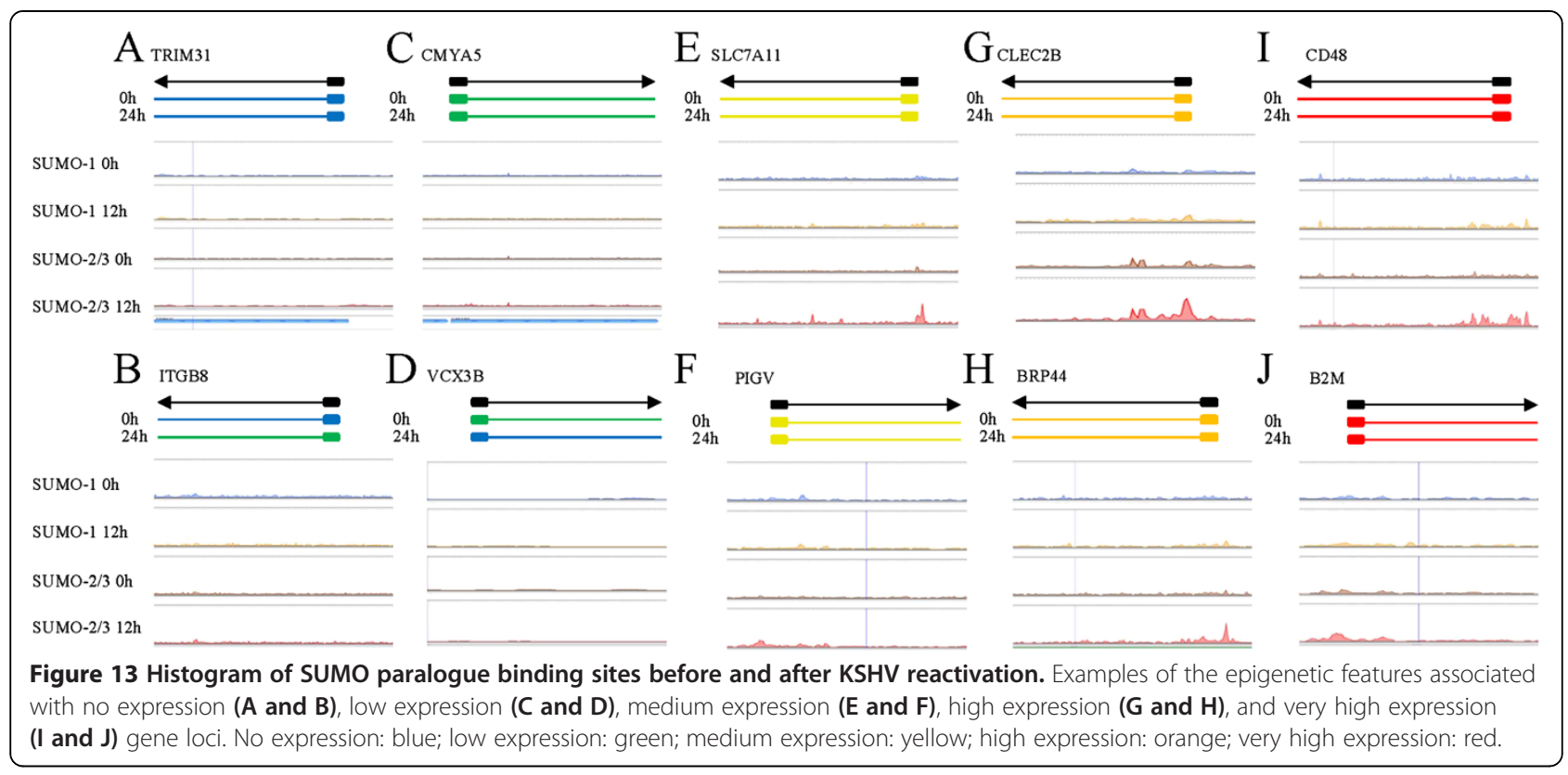

are preferentially localized on the promoters of highly expressed genes, and that SUMO-2/3 is predominantly found associated with highly expressed genes that show no change in expression during herpesvirus reactivation. Finally, after viral reactivation, SUMO-2/3 is significantly associated with the promoters of genes in pathways related to cellular immune responses, cytokine signaling, cell growth and apoptosis. To our knowledge, our findings are the first to compare dynamically the global chromatinbinding profiles of SUMO- 1 and SUMO-2/3 across the human genome and suggest that, while the binding profile of SUMO paralogues is similarly under un-induced condition, they do change differently during KSHV infection.

Herpesviruses have evolved multiple mechanisms to target SUMOylation pathways, including modulating SUMO conjugation enzymes (SUMO E1 ligase, SUMO E2 ligase and SUMO E3 ligase) and deconjugation enzymes (SUMO-specific proteases; SENP) as well as by directly targeting SUMOylated proteins [30]. Interestingly, KSHV encodes a SUMO E3 ligase in the lytic phase and this enzyme is likely to be the reason behind the increase in SUMO-2/3 paralogues present on chromatin during viral reactivation [16]. However, this hypothesis needs to be rigorously tested via a knock-in recombinant KSHV containing a SIM mutant of K-bZIP that results in a loss of its SUMO E3 ligase activity. This will be an interesting direction to investigate in the future. Moreover, we cannot exclude the possibility that the induction of K-Rta activates host SUMO E3 ligase to deposit SUMO-2/3 at the promoter regions. For example, we have previously identified a host factor, KAP1, is phosphorylated by KSHV vPK during KSHV reactivation [23] and KAP1 has recently been reported to be a SUMO E3 ligase for IRF-7 [52].

The complete sequence of the human genome was obtained more than a decade ago; nevertheless, our understanding of this genome is far from complete. The emerging concept from Encyclopedia of DNA Elements (ENCODE) is that biochemical functions of a genome can be assigned by systematically identifying the functional elements within the genome [53]. Patterns in chromatin modification or transcription factor binding onto the functional elements assists with the prediction of their role, particularly when RNA expression is examined. The global but uneven distribution of SUMO modification near TSSs prompted us to study the distribution of SUMO modification on different functional elements of the genome, such as promoters, coding sequences (transcripts), upstream gene regions, downstream gene regions, and intergenic regions. The significant enrichment of SUMO paralogues in promoter regions (Figure 2D and 2E) strongly suggests that SUMOylation may be involved in regulating gene transcription. Consistent with previous reports from lower eukaryotics and another describing SUMO-1 in HeLa cells $[7,46]$, the correlation between SUMO paralogues binding to promoter region and higher levels of gene transcription, which is also found in the present study (Figure 10), further supports the potential role of SUMOylation in maintaining the expression of constitutively active genes. Moreover, SUMO-1 and SUMO-2/3 may function in a similar manner maintaining the expression of transcriptional active genes in non-reactivated control cells. 


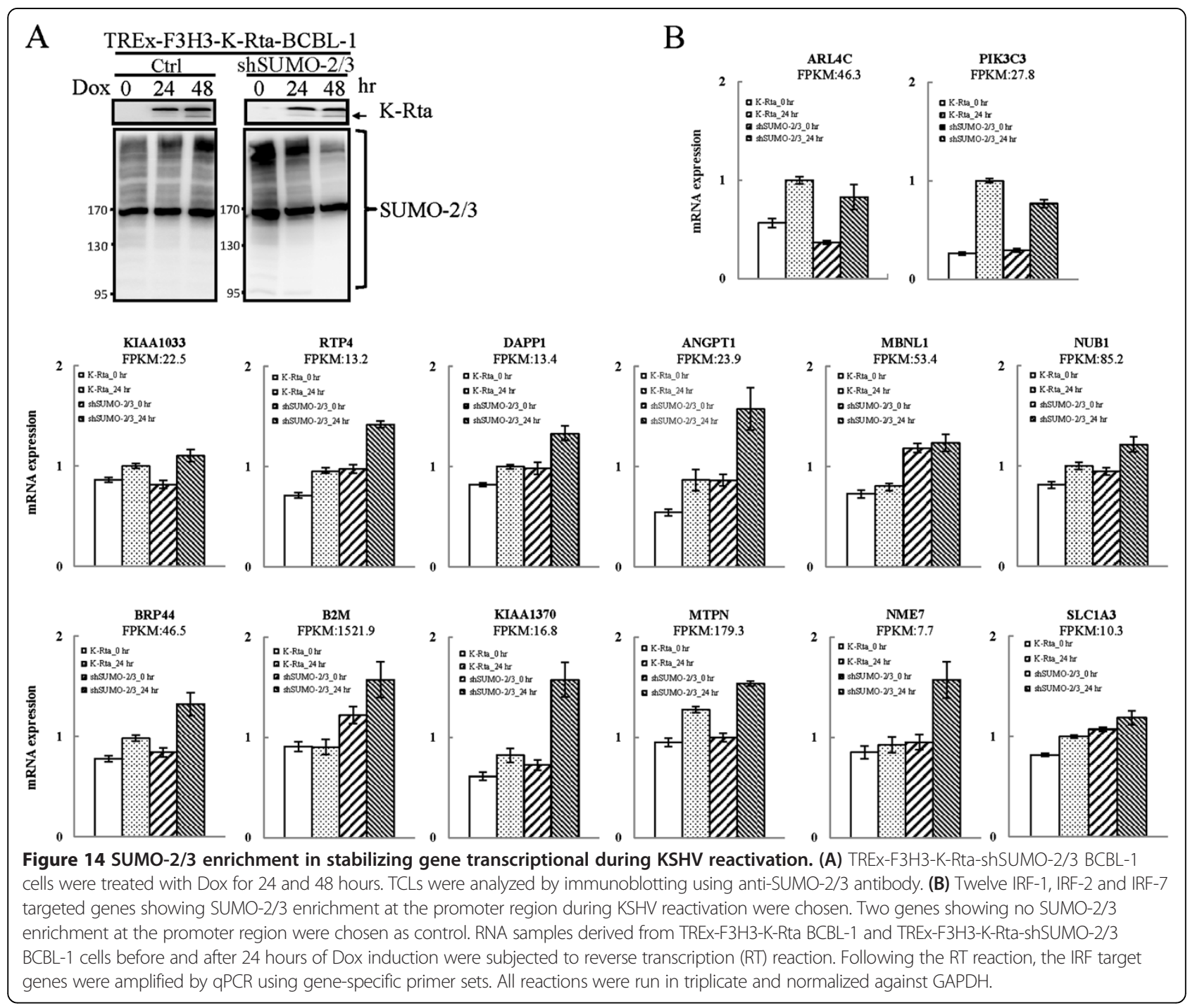

SUMO binding onto chromatin must occur via either the modification of chromatin remodeling proteins or the modification of transcription factors, both of which bind to the genome. SUMO shows focal peaks or areas of high occupancy within the promoter region near TSSs. The focal and gene-selective nature of SUMO occupancy resembles the peaks associated with transcription factors, which suggests that there is SUMO modification of TFs. Motif scanning is a powerful method to facilitate the identification of DNA binding motifs (or transcription factor binding motifs) from peaks defined by ChIP-seq. This method has been widely used to distinguish the transcription regulation of one or a few TFs. SUMO modifications are able to occur in many dozens of known TFs as well as being likely to occur in many currently unknown TFs. Using the current findings, it is probably too complex and too time consuming to carrying full scale motif scanning to identify potential SUMO target TFs. Therefore, as an alternative, we used an annotation method that directly annotates SUMO peaks in the promoter region in relation to transcription factor binding sites (TFBS). The details of this method have been submitted in another article [54]. Briefly, the Transfac Matrix Database (v7.0) created by Biobase contains 258 TFBS weight matrices representing the potential DNA binding sites of $176 \mathrm{TFs}$ and this database was chosen to annotate the SUMO peaks. Using this method, we were able to simultaneously identify potential SUMOylated TFs. Potential SUMO target TFs are those TFBSs that show a significant correlation with SUMO peaks and these were identified by the Hampel Identifier. Half of all SUMO-1 and half of the top-20 SUMO-2/3 potential target TFs identified before and after viral reactivation were known SUMO targets. The other half may be potential SUMO targets that have not been identified as yet or proteins 


\begin{tabular}{|c|c|c|c|c|c|c|c|c|c|c|}
\hline & \multirow[b]{3}{*}{ Pathway category } & \multirow[b]{3}{*}{$\begin{array}{l}\text { Genes in } \\
\text { category }\end{array}$} & \multicolumn{4}{|c|}{ SUMO-1 } & \multicolumn{4}{|c|}{ SUMO-2/3 } \\
\hline & & & \multicolumn{2}{|c|}{$\mathrm{Oh}$} & \multicolumn{2}{|c|}{$12 \mathrm{~h}$} & \multicolumn{2}{|c|}{$\mathrm{Oh}$} & \multicolumn{2}{|c|}{$12 \mathrm{~h}$} \\
\hline & & & $\begin{array}{c}\text { Genes } \\
\text { identified }\end{array}$ & $p$-value & $\begin{array}{l}\text { Genes } \\
\text { identified }\end{array}$ & $p$-value & $\begin{array}{l}\text { Genes } \\
\text { identified }\end{array}$ & $p$-value & $\begin{array}{l}\text { Genes } \\
\text { identified }\end{array}$ & $p$-value \\
\hline Pathogen-influenced signaling & Virus entry via endocytic pathways & 99 & 14 & $2.59 \mathrm{E}-01$ & 16 & $8.71 \mathrm{E}-02$ & 13 & $3.35 \mathrm{E}-01$ & 31 & $1.41 \mathrm{E}-03$ \\
\hline \multirow[t]{10}{*}{ Cellular immune response } & Antigen presentation pathway & 40 & 8 & $9.77 \mathrm{E}-02$ & 5 & $5.02 \mathrm{E}-01$ & 8 & $8.71 \mathrm{E}-02$ & 14 & $1.26 \mathrm{E}-02$ \\
\hline & CD40 signaling & 70 & 11 & 1.87E-01 & 12 & $8.91 \mathrm{E}-02$ & 11 & 1.67E-01 & 23 & $2.82 \mathrm{E}-03$ \\
\hline & HMGB1 signaling & 99 & 15 & 2.19E-01 & 18 & $3.89 \mathrm{E}-02$ & 13 & 3.96E-01 & 25 & 9.33E-02 \\
\hline & IL-10 signaling & 78 & 13 & $9.55 \mathrm{E}-02$ & 14 & 4.07E-02 & 10 & $3.58 \mathrm{E}-01$ & 19 & 9.55E-02 \\
\hline & IL-15 signaling & 67 & 8 & 5.43E-01 & 12 & 7.41E-02 & 8 & 5.15E-01 & 20 & $1.86 \mathrm{E}-02$ \\
\hline & IL-3 signaling & 74 & 11 & $3.11 \mathrm{E}-01$ & 12 & $1.74 \mathrm{E}-01$ & 10 & 4.11E-01 & 23 & $1.41 \mathrm{E}-02$ \\
\hline & IL-8 signaling & 205 & 29 & $1.52 \mathrm{E}-01$ & 28 & $1.61 \mathrm{E}-01$ & 29 & $1.25 \mathrm{E}-01$ & 48 & $4.68 \mathrm{E}-02$ \\
\hline & NF-kB activation by viruses & 82 & - & - & 12 & 1.99E-01 & - & - & 23 & $2.00 \mathrm{E}-02$ \\
\hline & p38 MAPK signaling & 117 & 22 & 4.47E-02 & 18 & 2.10E-01 & 17 & 3.10E-01 & 26 & 3.80E-01 \\
\hline & Toll-like receptor signaling & 62 & 14 & $1.05 \mathrm{E}-02$ & 9 & $2.65 \mathrm{E}-01$ & - & - & 17 & 5.75E-02 \\
\hline \multirow[t]{2}{*}{ Cytokine signaling } & IL-17A signaling in airway cells & 72 & 9 & 4.33E-01 & 12 & $8.91 \mathrm{E}-02$ & 10 & $2.72 \mathrm{E}-01$ & 21 & $1.35 \mathrm{E}-02$ \\
\hline & Prolactin signaling & 80 & 10 & $4.78 \mathrm{E}-01$ & 13 & $1.21 \mathrm{E}-01$ & - & - & 22 & $3.72 \mathrm{E}-02$ \\
\hline \multirow[t]{4}{*}{ Cancer } & Glioma signaling & 112 & 17 & $1.03 \mathrm{E}-01$ & 14 & $3.01 \mathrm{E}-01$ & - & - & 29 & $1.55 \mathrm{E}-02$ \\
\hline & p53 signaling & 96 & 18 & $5.13 \mathrm{E}-02$ & 16 & 1.17E-01 & 16 & $1.24 \mathrm{E}-01$ & 32 & $1.48 \mathrm{E}-03$ \\
\hline & Small cell lung cancer signaling & 89 & 13 & $1.24 \mathrm{E}-01$ & 13 & $1.02 \mathrm{E}-01$ & 13 & $1.08 \mathrm{E}-01$ & 27 & $6.03 E-04$ \\
\hline & Sonic hedgehog signaling & 33 & 6 & 1.65E-01 & 9 & $7.24 \mathrm{E}-03$ & 5 & 3.03E-01 & - & - \\
\hline \multirow{8}{*}{$\begin{array}{l}\text { Cellular growth, proliferation } \\
\text { and development }\end{array}$} & Cleavage and polyadenylation of Pre-mRNA & 12 & 5 & $1.32 \mathrm{E}-02$ & 4 & 5.37E-02 & 3 & 1.87E-01 & 5 & 8.32E-02 \\
\hline & EGF signaling & 62 & 11 & $1.02 \mathrm{E}-01$ & 8 & 4.05E-01 & - & - & 21 & $2.88 \mathrm{E}-03$ \\
\hline & FAK signaling & 101 & - & - & 15 & $1.15 \mathrm{E}-01$ & 12 & 4.06E-01 & 27 & $1.32 \mathrm{E}-02$ \\
\hline & JAK/Stat signaling & 70 & 13 & $8.71 \mathrm{E}-02$ & 13 & 7.08E-02 & 10 & 3.40E-01 & 26 & 5.37E-04 \\
\hline & Oncostatin M signaling & 35 & 5 & 4.55E-01 & 5 & 4.26E-01 & - & - & 12 & $3.55 \mathrm{E}-02$ \\
\hline & PDGF signaling & 85 & 12 & 2.92E-01 & 12 & $2.54 \mathrm{E}-01$ & - & - & 25 & 1.07E-02 \\
\hline & Thrombopoietin signaling & 63 & 8 & 4.22E-01 & 10 & 1.45E-01 & - & - & 17 & $4.90 \mathrm{E}-02$ \\
\hline & VEGF signaling & 103 & 17 & 7.59E-02 & 19 & $1.66 \mathrm{E}-02$ & 13 & 3.66E-01 & 25 & 7.59E-02 \\
\hline \multirow[t]{3}{*}{ Cell cycle regulation } & Cyclins and cell cycle regulation & 89 & 14 & $1.38 \mathrm{E}-01$ & 11 & 4.05E-01 & 15 & $6.92 \mathrm{E}-02$ & 24 & $2.75 \mathrm{E}-02$ \\
\hline & GADD45 signaling & 22 & 4 & $2.26 \mathrm{E}-01$ & 3 & 4.33E-01 & 5 & 8.32E-02 & 9 & 8.51E-03 \\
\hline & Mitotic roles of polo-like kinase & 70 & 11 & $2.46 \mathrm{E}-01$ & 11 & 2.14E-01 & 14 & 3.89E-02 & 18 & 1.39E-01 \\
\hline
\end{tabular}


Table 5 GO categories of SUMO targeting genes during KSHV reactivation (Continued)

\begin{tabular}{|c|c|c|c|c|c|c|c|c|c|c|}
\hline Growth factor signaling & ErbB2-ErbB3 signaling & 60 & - & - & 11 & $9.55 \mathrm{E}-02$ & - & - & 19 & $1.82 \mathrm{E}-02$ \\
\hline \multirow[t]{3}{*}{ Apoptosis } & April mediated signaling & 43 & 8 & $1.10 \mathrm{E}-01$ & 6 & $3.40 \mathrm{E}-01$ & 8 & $1.00 \mathrm{E}-01$ & 13 & $3.72 \mathrm{E}-02$ \\
\hline & Aryl hydrocarbon receptor signaling & 161 & 24 & $8.51 \mathrm{E}-02$ & 19 & $3.81 \mathrm{E}-01$ & 23 & $1.07 \mathrm{E}-01$ & 40 & $1.48 \mathrm{E}-02$ \\
\hline & Myc mediated apoptosis signaling & 60 & 10 & 2.13E-01 & 10 & 1.85E-01 & 9 & $3.10 \mathrm{E}-01$ & 20 & $1.05 \mathrm{E}-02$ \\
\hline Cellular stress and injury & Endoplasmic reticulum stress pathway & 18 & 7 & $5.25 \mathrm{E}-03$ & 5 & $6.61 \mathrm{E}-02$ & 5 & $6.76 \mathrm{E}-02$ & 7 & $6.03 \mathrm{E}-02$ \\
\hline Disease-specific pathways & Parkinson's signaling & 16 & 3 & $3.44 \mathrm{E}-01$ & 4 & $1.31 \mathrm{E}-01$ & 5 & 4.27E-02 & 4 & 4.32E-01 \\
\hline $\begin{array}{l}\text { Neurotransmitters and other } \\
\text { nervous system signaling }\end{array}$ & Cholecystokinin/gastrin-mediated signaling & 106 & 21 & $1.82 \mathrm{E}-02$ & 13 & 5.05E-01 & 15 & $2.94 \mathrm{E}-01$ & 24 & 2.59E-01 \\
\hline
\end{tabular}


Table 6 GO categories of SUMO targeting genes with no expression changes during KSHV reactivation

\begin{tabular}{|c|c|c|c|c|c|c|c|c|}
\hline & \multirow[b]{3}{*}{ Pathway category } & \multirow[b]{3}{*}{$\begin{array}{l}\text { Genes in } \\
\text { category }\end{array}$} & \multicolumn{4}{|c|}{ SUMO-2/3 $12 \mathrm{~h}$} & \multirow{2}{*}{\multicolumn{2}{|c|}{$\begin{array}{l}\text { SUMO-2/3 enriched at } 12 \mathrm{hr} \\
\text { Gene expression no change }\end{array}$}} \\
\hline & & & \multicolumn{2}{|c|}{ All genes } & \multicolumn{2}{|c|}{ Gene expression no change } & & \\
\hline & & & $\begin{array}{c}\text { Genes } \\
\text { identified }\end{array}$ & $p$-value & $\begin{array}{c}\text { Genes } \\
\text { identified }\end{array}$ & $p$-value & $\begin{array}{c}\text { Genes } \\
\text { identified }\end{array}$ & $p$-value \\
\hline Pathogen-influenced signaling & Virus entry via endocytic pathways & 99 & 31 & $1.41 \mathrm{E}-03$ & 28 & $3.24 \mathrm{E}-03$ & 20 & $5.13 \mathrm{E}-03$ \\
\hline \multirow[t]{10}{*}{ Cellular immune response } & Antigen Presentation pathway & 40 & 14 & $1.26 \mathrm{E}-02$ & 12 & $3.63 \mathrm{E}-02$ & 9 & $3.24 \mathrm{E}-02$ \\
\hline & CD40 signaling & 70 & 23 & $2.82 \mathrm{E}-03$ & 21 & $4.79 \mathrm{E}-03$ & 14 & $1.91 \mathrm{E}-02$ \\
\hline & HMGB1 signaling & 99 & 25 & $9.33 \mathrm{E}-02$ & 24 & $6.46 \mathrm{E}-02$ & 16 & $1.03 \mathrm{E}-01$ \\
\hline & IL-10 signaling & 78 & 19 & $9.55 \mathrm{E}-02$ & 16 & $2.07 \mathrm{E}-01$ & 11 & $2.10 \mathrm{E}-01$ \\
\hline & IL-15 signaling & 67 & 20 & $1.86 \mathrm{E}-02$ & 19 & $1.55 \mathrm{E}-02$ & 16 & $2.34 \mathrm{E}-03$ \\
\hline & IL-3 signaling & 74 & 23 & $1.41 \mathrm{E}-02$ & 22 & $1.02 \mathrm{E}-02$ & 14 & $4.90 \mathrm{E}-02$ \\
\hline & IL-8 signaling & 205 & 48 & $4.68 \mathrm{E}-02$ & 44 & $6.92 \mathrm{E}-02$ & 27 & $2.14 \mathrm{E}-01$ \\
\hline & $\mathrm{NF}-\mathrm{kB}$ activation by viruses & 82 & 23 & $2.00 \mathrm{E}-02$ & 21 & $3.16 \mathrm{E}-02$ & 15 & $3.39 \mathrm{E}-02$ \\
\hline & p38 MAPK signaling & 117 & 26 & $3.80 \mathrm{E}-01$ & 24 & $3.88 \mathrm{E}-01$ & 16 & $3.79 \mathrm{E}-01$ \\
\hline & Toll-like receptor signaling & 62 & 17 & $5.75 \mathrm{E}-02$ & 15 & $9.55 \mathrm{E}-02$ & 11 & $7.76 \mathrm{E}-02$ \\
\hline \multirow[t]{2}{*}{ Cytokine signaling } & IL-17A signaling in airway cells & 72 & 21 & $1.35 \mathrm{E}-02$ & 2 & $8.51 \mathrm{E}-02$ & 11 & $1.58 \mathrm{E}-01$ \\
\hline & Prolactin signaling & 80 & 22 & $3.72 \mathrm{E}-02$ & 22 & $1.45 \mathrm{E}-02$ & 17 & $6.46 \mathrm{E}-03$ \\
\hline \multirow[t]{3}{*}{ Cancer } & Glioma signaling & 112 & 29 & $1.55 \mathrm{E}-02$ & 27 & $1.62 \mathrm{E}-02$ & 19 & $2.14 \mathrm{E}-02$ \\
\hline & p53 signaling & 96 & 32 & $1.48 \mathrm{E}-03$ & 27 & $1.23 \mathrm{E}-02$ & 20 & $8.51 E-03$ \\
\hline & Small cell lung cancer signaling & 89 & 27 & $6.03 \mathrm{E}-04$ & 26 & $3.63 \mathrm{E}-04$ & 20 & $2.57 \mathrm{E}-04$ \\
\hline \multirow[t]{8}{*}{ Cellular growth, proliferation and development } & Cleavage and polyadenylation of pre-mRNA & 12 & 5 & $8.32 \mathrm{E}-02$ & 5 & $6.03 \mathrm{E}-02$ & 4 & $5.01 \mathrm{E}-02$ \\
\hline & EGF signaling & 62 & 21 & $2.88 \mathrm{E}-03$ & 20 & $2.34 \mathrm{E}-03$ & 13 & $1.66 \mathrm{E}-02$ \\
\hline & FAK signaling & 101 & 27 & $1.32 \mathrm{E}-02$ & 26 & $9.33 \mathrm{E}-03$ & 17 & 3.47E-02 \\
\hline & JAK/Stat signaling & 70 & 26 & $5.37 \mathrm{E}-04$ & 24 & $8.71 \mathrm{E}-04$ & 20 & $1.07 \mathrm{E}-04$ \\
\hline & Oncostatin M signaling & 35 & 12 & $3.55 \mathrm{E}-02$ & 11 & 4.47E-02 & 8 & $5.01 \mathrm{E}-02$ \\
\hline & PDGF signaling & 85 & 25 & $1.07 \mathrm{E}-02$ & 24 & $6.92 \mathrm{E}-03$ & 18 & $5.01 \mathrm{E}-03$ \\
\hline & Thrombopoietin signaling & 63 & 17 & $4.90 \mathrm{E}-02$ & 17 & $2.29 \mathrm{E}-02$ & 12 & $3.31 E-02$ \\
\hline & VEGF signaling & 103 & 25 & $7.59 \mathrm{E}-02$ & 25 & 3.47E-02 & 16 & $9.55 \mathrm{E}-02$ \\
\hline \multirow[t]{3}{*}{ Cell cycle regulation } & Cyclins and cell cycle regulation & 89 & 24 & $2.75 \mathrm{E}-02$ & 23 & $2.19 \mathrm{E}-02$ & 13 & $1.80 \mathrm{E}-01$ \\
\hline & GADD45 signaling & 22 & 9 & $8.51 \mathrm{E}-03$ & 8 & $2.40 \mathrm{E}-02$ & 4 & $2.26 \mathrm{E}-01$ \\
\hline & Mitotic roles of polo-like kinase & 70 & 18 & $1.39 \mathrm{E}-01$ & 18 & $7.24 \mathrm{E}-02$ & 9 & 4.44E-01 \\
\hline Growth Ffactor signaling & ErbB2-ErbB3 signaling & 60 & 19 & $1.82 \mathrm{E}-02$ & 19 & $7.08 \mathrm{E}-03$ & 15 & $3.02 \mathrm{E}-03$ \\
\hline \multirow[t]{3}{*}{ Apoptosis } & April mediated signaling & 43 & 13 & $3.72 \mathrm{E}-02$ & 11 & $9.12 \mathrm{E}-02$ & 6 & $3.24 \mathrm{E}-01$ \\
\hline & Aryl hydrocarbon receptor signaling & 161 & 40 & $1.48 \mathrm{E}-02$ & 34 & $6.92 \mathrm{E}-02$ & 21 & $1.96 \mathrm{E}-01$ \\
\hline & Myc mediated apoptosis signaling & 60 & 20 & $1.05 \mathrm{E}-02$ & 19 & $8.71 \mathrm{E}-03$ & 14 & $9.33 \mathrm{E}-03$ \\
\hline Cellular stress and injury & Endoplasmic reticulum stress pathway & 18 & 7 & $6.03 \mathrm{E}-02$ & 7 & $3.98 \mathrm{E}-02$ & 4 & $1.73 \mathrm{E}-01$ \\
\hline Disease-specific pathways & Parkinson's signaling & 16 & 4 & $4.32 \mathrm{E}-01$ & 4 & $3.61 \mathrm{E}-01$ & - & \\
\hline Neurotransmitters and other nervous system signaling & Cholecystokinin/gastrin-mediated signaling & 106 & 24 & $2.59 \mathrm{E}-01$ & 20 & 4.56E-01 & - & \\
\hline
\end{tabular}


containing a SIM domain that provides an additional interaction platform allowing the recruiting of other SUMOylated proteins; both of these situations may be responsible for the TFs identified here. The SUMOylation fraction in a steady state is typically very little in related to the entire pool of transcription factors. Efforts are still needed to confirm the results outlined here and to elucidate the underlying functions of SUMOylation during the regulation of these TFs. Interestingly, when we ranked the potential SUMO-2/3 target TFs by the total number of their regulating genes (Figures 4 and 5), we found three IRFs that were not SUMO-2/3 targets in the control cells that were listed as top 4th, top 5th and top 6th of the SUMO-2/3 target TFs after viral reactivation. IRFs constitute a family of TFs (IRF-1-IRF-9) that are in control of the type I interferon (IFN) system and are involved in executing the innate and adaptive immunity associated with host resistance against pathogens, including virus infection. To promote its own survival, KSHV exploits a number of different strategies to suppress the host immune system. Recent evidence has shown that the virus triggers the SUMOylation of IRFs, leading to a targeting and blocking of the type I interferon pathway [24,40,41]. K-bZIP of KSHV has also been found to inhibit type I IFN signaling in a signal transducers and activators of transcription (STAT) dependent manner and in an IFN-stimulated gene factor 3 (ISGF3) independent manner [39]. Moreover, KSHV K-bZIP inhibits IRF-3 by preventing IRF-3 from binding to target promoter, which precludes the formation of the enhanceosome. The potential SUMO-2/3 target IRFs identified here (Figure 5) provides an additional novel mechanism for globally inhibiting the activation of the host immune system.

The growing links between the viral and cellular SUMO systems makes SUMO a potential target for antiviral therapy [21]. Identifying the preferential usage of SUMO paralogues in viruses may help to improve the specificity of any SUMO-targeted antiviral therapies. Recently, growing evidence, including ours, suggests that some herpesviruses have a preference for SUMO-2/3 [16,55]. Significant increase in SUMO-2/3 coating across human genome, but not in SUMO-1 coating, during viral reactivation found here suggest that a new class of combine therapy targeting SUMO-2/3 may disrupt the dynamic balance of the herpesvirus latent and lytic phases. Disrupting the balance may help the clearance of the herpesvirus from the infected cells and improve current therapy.

In summary, we found that SUMO- 1 and SUMO-2/3 share a highly similar binding landscape on chromatin. They are preferentially enriched in promoter regions and are associated with highly transcribed genes. Differential chromatin-binding profiles of the SUMO paralogues are able to be observed during herpesvirus reactivation. We found that SUMO-2/3 peaks significantly increased in promoter regions during viral reactivation and this was associated with the genes that do not undergo changes in transcription level. TFs identification and GO analysis suggests that SUMO-2/3 preferentially target immune pathways during viral reactivation.

\section{Competing interests}

The authors disclose no potential conflicts of interest.

\section{Authors' contributions}

PCC, CYC and HJK conceived the project and wrote the paper. CYC, CHC, and WPH participated in the ChIP-seq alignment and performed the statistical analysis. CYC, CHC, TYC, and YWL participated in the RNA-seq alignment and performed the statistical analysis. CYT, HWW, and WCW provided conceptual advice. PCC, CLH, and SML participated in ChIP-Seq experiments. PCC, MC, YCY, and HWH performed the ChIP assay, RNA preparation, IP, immunoblotting, ChIP-qPCR and RT-qPCR experiments. PCC, CYC and YCY performed the identification of SUMO targeting TF and the statistical analysis. PCC and YCY performed ChIP-seq versus RNA-Seq and GO analysis. All authors read and approved the final manuscript.

Authors' information

Chia-Yang Cheng is the co-first author.

\section{Acknowledgements}

The work was supported by NSC grants (101-2321-B-010-017) and by NHRI grants (NHRI-EX102-10215BC). We thank the Biomedical Science and Engineering Center at National Tsing Hua University for the ChIP-Seq experiments and the Sequencing Core of National Research Program for Genomic Medicine at National Yang Ming University VYM Genome Research Center for the RNA-Seq experiments.

\section{Author details}

${ }^{1}$ Institute of Microbiology and Immunology, National Yang-Ming University, Taipei 11221, Taiwan. ${ }^{2}$ Institute of Molecular and Cellular Biology and Department of Life Science, National Tsing Hua University, Hsinchu 300, Taiwan. ${ }^{3}$ Department of Computer Science, National Tsing Hua University, Hsinchu 300, Taiwan. ${ }^{4}$ UC Davis Cancer Center, University of California, Davis, CA 95616, USA. ${ }^{5}$ Division of Molecular and Genomic Medicine, National Health Research Institutes, 35 Keyan Road, Zhunan, Miaoli County 35053, Taiwan. ${ }^{6}$ Department of Biochemistry and Molecular Medicine, University of California, Davis, CA 95616, USA. ${ }^{7}$ Institute of Statistics, National Tsing Hua University, Hsinchu 300, Taiwan. ${ }^{8}$ Institute for Translational Medicine, College of Medical Science and Technology, Taipei Medical University, 250 Wu-Xin Street, Taipei City, Taiwan.

Received: 15 July 2013 Accepted: 19 November 2013

Published: 23 November 2013

\section{References}

1. Lomeli $H$, Vazquez M: Emerging roles of the SUMO pathway in development. Cell Mol Life Sci 2011, 68:4045-4064.

2. Nacerddine K, Lehembre F, Bhaumik M, Artus J, Cohen-Tannoudji M, Babinet C, Pandolfi PP, Dejean A: The SUMO pathway is essential for nuclear integrity and chromosome segregation in mice. Dev Cell 2005, 9:769-779.

3. Prudden J, Perry JJ, Nie M, Vashisht AA, Arvai AS, Hitomi C, Guenther G, Wohlschlegel JA, Tainer JA, Boddy MN: DNA repair and global sumoylation are regulated by distinct Ubc9 noncovalent complexes. Mol Cell Biol 2011, 31:2299-2310.

4. Garcia-Dominguez M, Reyes JC: SUMO association with repressor complexes, emerging routes for transcriptional control. Biochim Biophys Acta 2009, 1789:451-459.

5. Wilkinson KA, Henley JM: Mechanisms, regulation and consequences of protein SUMOylation. Biochem J 2010, 428:133-145.

6. Maison C, Bailly D, Roche D, Montes de Oca R, Probst AV, Vassias I, Dingli F, Lombard B, Loew D, Quivy JP, Almouzni G: SUMOylation promotes de novo targeting of HP1alpha to pericentric heterochromatin. Nat Genet 2011, 43:220-227. 
7. Rosonina E, Duncan SM, Manley JL: SUMO functions in constitutive transcription and during activation of inducible genes in yeast. Genes Dev 2010, 24:1242-1252

8. Makhnevych T, Sydorskyy Y, Xin X, Srikumar T, Vizeacoumar FJ, Jeram SM, Li Z, Bahr S, Andrews BJ, Boone C, Raught B: Global map of SUMO function revealed by protein-protein interaction and genetic networks. Mol Cell 2009, 33:124-135.

9. Tatham $\mathrm{MH}$, Jaffray $\mathrm{E}$, Vaughan $\mathrm{OA}$, Desterro JM, Botting $\mathrm{CH}$, Naismith $\mathrm{JH}$, Hay RT: Polymeric chains of SUMO-2 and SUMO-3 are conjugated to protein substrates by SAE1/SAE2 and Ubc9. J Bio/ Chem 2001, 276:35368-35374.

10. Matic I, van Hagen M, Schimmel J, Macek B, Ogg SC, Tatham MH, Hay RT, Lamond Al, Mann M, Vertegaal AC: In vivo identification of human small ubiquitin-like modifier polymerization sites by high accuracy mass spectrometry and an in vitro to in vivo strategy. Mol Cell Proteomics 2008, 7:132-144.

11. Saitoh $H$, Hinchey J: Functional heterogeneity of small ubiquitin-related protein modifiers SUMO-1 versus SUMO-2/3. J Biol Chem 2000, 275:6252-6258.

12. Ayaydin F, Dasso M: Distinct in vivo dynamics of vertebrate SUMO paralogues. Mol Biol Cell 2004, 15:5208-5218.

13. Kolli N, Mikolajczyk J, Drag M, Mukhopadhyay D, Moffatt N, Dasso M, Salvesen G, Wilkinson KD: Distribution and paralogue specificity of mammalian deSUMOylating enzymes. Biochem J 2010, 430:335-344.

14. Lin DY, Huang YS, Jeng JC, Kuo HY, Chang CC, Chao T, Ho CC, Chen YC, Lin TP, Fang HI, et al: Role of SUMO-interacting motif in Daxx SUMO modification, subnuclear localization, and repression of sumoylated transcription factors. Mol Cell 2006, 24:341-354.

15. Namanja AT, Li YJ, Su Y, Wong S, Lu J, Colson LT, Wu C, Li SS, Chen Y: Insights into high affinity small ubiquitin-like modifier (SUMO) recognition by SUMO-interacting motifs (SIMs) revealed by a combination of NMR and peptide array analysis. J Bio/ Chem 2012, 287:3231-3240

16. Chang PC, Izumiya Y, Wu CY, Fitzgerald LD, Campbell M, Ellison TJ, Lam KS, Luciw PA, Kung HJ: Kaposi's sarcoma-associated herpesvirus (KSHV) encodes a SUMO E3 ligase that is SIM-dependent and SUMO-2/3specific. J Biol Chem 2010, 285:5266-5273.

17. Gareau JR, Reverter D, Lima CD: Determinants of small ubiquitin-like modifier 1 (SUMO1) protein specificity, E3 ligase, and SUMO-RanGAP1 binding activities of nucleoporin RanBP2. J Biol Chem 2012, 287:4740-4751.

18. Tatham MH, Kim S, Jaffray E, Song J, Chen Y, Hay RT: Unique binding interactions among Ubc9, SUMO and RanBP2 reveal a mechanism for SUMO paralog selection. Nat Struct Mol Biol 2005, 12:67-74.

19. Meulmeester E, Kunze M, Hsiao HH, Urlaub H, Melchior F: Mechanism and consequences for paralog-specific sumoylation of ubiquitin-specific protease 25. Mol Cell 2008, 30:610-619.

20. Bailey D, O'Hare P: Herpes simplex virus 1 ICPO co-localizes with a SUMOspecific protease. J Gen Virol 2002, 83:2951-2964.

21. Boggio R, Chiocca S: Viruses and sumoylation: recent highlights. Curr Opin Microbiol 2006, 9:430-436.

22. Boggio R, Colombo R, Hay RT, Draetta GF, Chiocca S: A mechanism for inhibiting the SUMO pathway. Mol Cell 2004, 16:549-561.

23. Chang PC, Fitzgerald LD, Van Geelen A, Izumiya Y, Ellison TJ, Wang DH, Ann DK, Luciw PA, Kung HJ: Kruppel-associated box domain-associated protein-1 as a latency regulator for Kaposi's sarcoma-associated herpesvirus and its modulation by the viral protein kinase. Cancer Res 2009, 69:5681-5689.

24. Chang TH, Kubota T, Matsuoka M, Jones S, Bradfute SB, Bray M, Ozato K: Ebola Zaire virus blocks type I interferon production by exploiting the host SUMO modification machinery. PLoS Pathog 2009, 5:e1000493.

25. Izumiya Y, Ellison TJ, Yeh ET, Jung JU, Luciw PA, Kung HJ: Kaposi's sarcomaassociated herpesvirus K-bZIP represses gene transcription via SUMO modification. J Virol 2005, 79:9912-9925.

26. Adamson AL, Kenney S: Epstein-barr virus immediate-early protein BZLF is SUMO-1 modified and disrupts promyelocytic leukemia bodies. J Virol 2001, 75:2388-2399.

27. Chang LK, Lee YH, Cheng TS, Hong YR, Lu PJ, Wang JJ, Wang WH, Kuo CW Li SS, Liu ST: Post-translational modification of Rta of Epstein-Barr virus by SUMO-1. J Biol Chem 2004, 279:38803-38812

28. Chang LK, Liu ST, Kuo CW, Wang WH, Chuang JY, Bianchi E, Hong YR: Enhancement of transactivation activity of Rta of Epstein-Barr virus by RanBPM. J Mol Biol 2008, 379:231-242.

29. Hagemeier SR, Dickerson SJ, Meng Q, Yu X, Mertz JE, Kenney SC: Sumoylation of the Epstein-Barr virus BZLF1 protein inhibits its transcriptional activity and is regulated by the virus-encoded protein kinase. J Virol 2010, 84:4383-4394.
30. Wimmer $P$, Schreiner $S$, Dobner T: Human pathogens and the host cell SUMOylation system. J Virol 2012, 86:642-654

31. Wen KW, Damania B: Kaposi sarcoma-associated herpesvirus (KSHV) molecular biology and oncogenesis. Cancer Lett 2010, 289:140-150.

32. Moore PS, Chang Y: Why do viruses cause cancer? Highlights of the first century of human tumour virology. Nat Rev Cancer 2010, 10:878-889.

33. Chang Y, Moore PS: Kaposi's Sarcoma (KS)-associated herpesvirus and its role in KS. Infect Agents Dis 1996, 5:215-222

34. Boshoff C, Schulz TF, Kennedy MM, Graham AK, Fisher C, Thomas A, McGee JO, Weiss RA, O'Leary JJ: Kaposi's sarcoma-associated herpesvirus infects endothelial and spindle cells. Nat Med 1995, 1:1274-1278.

35. Coscoy L: Immune evasion by Kaposi's sarcoma-associated herpesvirus. Nat Rev Immunol 2007, 7:391-401.

36. Lee HR, Brulois $K$, Wong $L$, Jung JU: Modulation of immune system by Kaposi's sarcoma-associated herpesvirus: lessons from viral evasion strategies. Front Microbiol 2012, 3:44.

37. Lefort S, Soucy-Faulkner A, Grandvaux N, Flamand L: Binding of Kaposi's sarcoma-associated herpesvirus K-bZIP to interferon-responsive factor 3 elements modulates antiviral gene expression. J Virol 2007, 81:10950-10960.

38. Yu Y, Wang SE, Hayward GS: The KSHV immediate-early transcription factor RTA encodes ubiquitin E3 ligase activity that targets IRF7 for proteosome-mediated degradation. Immunity 2005, 22:59-70.

39. Lefort S, Gravel A, Flamand L: Repression of interferon-alpha stimulated genes expression by Kaposi's sarcoma-associated herpesvirus K-bZIP protein. Virology 2010, 408:14-30.

40. Bentz GL, Shackelford J, Pagano JS: Epstein-Barr virus latent membrane protein 1 regulates the function of interferon regulatory factor 7 by inducing its sumoylation. J Virol 2012, 86:12251-12261.

41. Kubota T, Matsuoka M, Chang TH, Tailor P, Sasaki T, Tashiro M, Kato A, Ozato K: Virus infection triggers SUMOylation of IRF3 and IRF7, leading to the negative regulation of type I interferon gene expression. J Biol Chem 2008, 283:25660-25670.

42. Richner JM, Clyde K, Pezda AC, Cheng BY, Wang T, Kumar GR, Covarrubias S, Coscoy L, Glaunsinger B: Global mRNA degradation during lytic gammaherpesvirus infection contributes to establishment of vira latency. PLoS Pathog 2011, 7:e1002150.

43. Smiley JR: Herpes simplex virus virion host shutoff protein: immune evasion mediated by a viral RNase? J Virol 2004, 78:1063-1068.

44. Covarrubias S, Gaglia MM, Kumar GR, Wong W, Jackson AO, Glaunsinger BA: Coordinated destruction of cellular messages in translation complexes by the gammaherpesvirus host shutoff factor and the mammalian exonuclease Xrn1. PLoS Pathog 2011, 7:e1002339.

45. Chang PC, Fitzgerald LD, Hsia DA, Izumiya Y, Wu CY, Hsieh WP, Lin SF, Campbell M, Lam KS, Luciw PA, et al: Histone demethylase JMJD2A regulates Kaposi's sarcoma-associated herpesvirus replication and is targeted by a viral transcriptional factor. J Virol 2011, 85:3283-3293.

46. Liu HW, Zhang J, Heine GF, Arora M, Gulcin Ozer H, Onti-Srinivasan R, Huang K, Parvin JD: Chromatin modification by SUMO-1 stimulates the promoters of translation machinery genes. Nucleic Acids Res 2012, 40:10172-10186.

47. Chang CC, Naik MT, Huang YS, Jeng JC, Liao PH, Kuo HY, Ho CC, Hsieh YL, Lin $\mathrm{CH}$, Huang NJ, et al: Structural and functional roles of Daxx SIM phosphorylation in SUMO paralog-selective binding and apoptosis modulation. Mol Cell 2011, 42:62-74.

48. Lyst MJ, Stancheva I: A role for SUMO modification in transcriptional repression and activation. Biochem Soc Trans 2007, 35:1389-1392.

49. Davies L, Gather U: The identification of multiple outliers. J Am Stat Assoc 1993, 88:782-792.

50. Stielow B, Sapetschnig A, Kruger I, Kunert N, Brehm A, Boutros M, Suske G: Identification of SUMO-dependent chromatin-associated transcriptional repression components by a genome-wide RNAi screen. Mol Cell 2008 29:742-754

51. Zhang XD, Goeres J, Zhang H, Yen TJ, Porter AC, Matunis MJ: SUMO-2/3 modification and binding regulate the association of CENP-E with kinetochores and progression through mitosis. Mol Cell 2008, 29:729-741.

52. Liang Q, Deng H, Li X, Wu X, Tang Q, Chang TH, Peng H, Rauscher FJ 3rd, Ozato K, Zhu F: Tripartite motif-containing protein 28 is a small ubiquitinrelated modifier E3 ligase and negative regulator of IFN regulatory factor 7. J Immunol 2011, 187:4754-4763.

53. Dunham I, Kundaje A, Aldred SF, Collins PJ, Davis CA, Doyle F, Epstein CB, Frietze S, Harrow J, Kaul R, et al: An integrated encyclopedia of DNA elements in the human genome. Nature 2012, 489:57-74. 
54. Cheng $\mathrm{CY}$, Chu CH, Hsu HW, Hsu FR, Tang CY, Wang WC, Kung HJ, Chang PC: An improved ChIP-seq peak detection system for simultaneously identifying post-translational modified transcription factors by combinatorial fusion, using SUMOylation as an example. APBC2014 (Accepted).

55. Marusic MB, Mencin N, Licen M, Banks L, Grm HS: Modification of human papillomavirus minor capsid protein L2 by sumoylation. J Virol 2010, 84:11585-11589.

doi:10.1186/1471-2164-14-824

Cite this article as: Chang et al:: The chromatin modification by SUMO-2/3

but not SUMO-1 prevents the epigenetic activation of key immune-related genes during Kaposi's sarcoma associated herpesvirus reactivation. BMC Genomics 2013 14:824

\section{Submit your next manuscript to BioMed Central and take full advantage of:}

- Convenient online submission

- Thorough peer review

- No space constraints or color figure charges

- Immediate publication on acceptance

- Inclusion in PubMed, CAS, Scopus and Google Scholar

- Research which is freely available for redistribution 